\title{
An assessment of the $J$-integral test for a metallic foam
}

\author{
H. C. Tankasala ${ }^{\text {a }}$, T. Li ${ }^{\text {a }}$, P. E. Seiler ${ }^{\text {a }}$, V. S. Deshpande ${ }^{\text {a }}$, N. A. Fleck ${ }^{\mathrm{a}, *}$ \\ a Department of Engineering, University of Cambridge,Cambridge CB2 1PZ, United Kingdom
}

\begin{abstract}
An assessment is made of the J-integral test procedure for initial crack growth in an opencell aluminium alloy foam by combining finite element $(\mathrm{FE})$ simulations with experiment. It is found experimentally that a zone of randomly failed struts develops ahead of the primary crack tip, and is comparable in size to that of the plastic zone. Hence, a crack tip J-field is absent at the initiation of crack growth from the primary crack tip. This implies that the measured $J_{\text {IC }}$ value and the $J$ versus crack extension $\Delta a$ curve cannot be treated as material properties despite the fact that the specimen size meets the usual criteria for $J$ validity. The toughness tests were performed on a single-edge notched bend specimen, and crack extension was measured by the direct current potential drop method, by digital image correlation and by X-ray computed tomography. The crack growth resistance of the foam is associated with two distinct zones of plastic dissipation: (i) a bulk plastic zone emanating from the crack tip (containing a cluster of randomly failed struts), and (ii) a crack bridging zone behind the advancing crack tip. The applicability of a cohesive zone model to predict the fracture response is explored for the observed case of large scale bridging. To do so, FE simulations are performed by replacing the discrete lattice of the open-cell metallic foam by a compressible, elastic-plastic hardening solid while the fracture process zone in the foam is represented by a cohesive zone, as characterised by a tensile traction versus separation law. A detailed comparison of the cohesive zone model with experimental observations reveals that it is possible to capture the load versus displacement response but not the details of the fracture process zone using a single set of process zone parameters.
\end{abstract}

Keywords: metal foams, fracture toughness, bridging zone, cohesive zone model

\footnotetext{
${ }^{*}$ Corresponding author

Email addresses: hct30@cam. ac.uk (H. C. Tankasala), naf 1@eng.cam.ac.uk (N. A. Fleck)
} 


\section{Introduction}

Metallic foams enjoy increasing application in sandwich panels for lightweight structural components, in energy absorption systems for protection from impacts, in heat sinks for electronic devices and in acoustic insulation, inter alia. The focus of this study is the fracture response of open-cell aluminium alloy foams.

The standard method for measuring the fracture toughness and crack growth resistance of ductile materials, including metallic foams, is the $J$-integral procedure as outlined in the ASTM standard E1820 [? ], see for example ? ] for a review of fracture toughness tests on porous materials. The ability of the J-integral to characterise crack initiation and crack growth is predicated upon the existence of a near-tip J-field, such as the HRR field in a fully dense elasto-plastic solid. This J-field must encompass the fracture process zone (FPZ) at the crack tip, as sketched in Fig. 1(a). Recall that the FPZ is on the order of the crack tip opening displacement for a fully dense metallic solid. At issue is whether the FPZ much exceeds the cell size $\ell$ of a metallic foam, particularly when failure is stochastic in nature and involves the failure of struts in a zone ahead of the crack tip.

Previous studies of the fracture of metallic foams assume that a crack bridging zone develops only after advance of the main crack tip, with successive strut-by-strut failure occurring at the crack tip [? ? ? . However, this assumption needs to be validated by experiment. It may be that a large FPZ develops ahead of the main crack tip in metallic foams, with a stochastic distribution of strut failure in existence before the main crack tip advances. If this FPZ is comparable in size to the plastic zone size, as illustrated in Fig. 1(b), then no J-field exists, regardless of the overall size of the specimen and of the initial crack length. The relative size of FPZ to plastic zone is unclear from the literature on metallic foams, and is measured in the present study.

? ] have performed J-tests on compact tension specimens made from aluminium-replicated open-cell foams. Their open-cell foams have a small and relatively uniform cell diameter 
$(\approx 400 \mu \mathrm{m})$ and are made from highly ductile pure aluminium. They observed that strut fracture was stochastic in a zone ahead of the crack tip, and the resulting crack extension is accompanied by the development of a substantial crack-bridging zone. For example, their bend specimen had a net section ligament of length $10 \mathrm{~mm}$, ahead of the pre-crack, at the start of the toughness test, and they report a large scale bridging zone of length $5 \mathrm{~mm}$ behind the crack tip after crack advance on the order of $7 \mathrm{~mm}$. Hence, it is unlikely that a J-field surrounds the advancing crack tip. Similar observations on the existence of a bridging zone (of size 4 to 8 cells) in the wake of an advancing crack have been reported for the case of open-cell aluminium alloy foams [? ], open-cell titanium alloy foams [? ], and closed-cell aluminium alloy foams [? ? ]. These experimental studies all followed the ASTM standard E1820 [?] to measure the crack growth resistance from the fracture response of a bend specimen, and each met the size criteria as demanded by the standard. A significant $R$-curve behaviour is reported in each of these studies due to the presence of crack bridging ligaments behind the advancing crack tip. ? ] have used the same ASTM procedure for measuring the crack growth resistance of 3D octet-truss lattices. They observed that the fracture toughness scales linearly with the square root of the cell size and linearly with the lattice relative density. However, in contrast to the fracture process observed in foams, the struts of the lattice failed cell by cell at the crack tip, without the formation of a recognisable bridging zone.

\subsection{Prediction of crack growth in metallic foams}

The cohesive zone approach is a useful predictive tool for modelling crack growth in the presence of bulk plasticity as observed in metallic foams. In this approach, the details of the crack tip failure mechanism are not stated explicitly. Rather, a cohesive zone, as defined by a tensile traction versus separation response, idealises the FPZ at the crack tip and is embedded within an elasto-plastic continuum. The cohesive zone approach has the virtue that it can be used to model both small scale and large scale bridging [? ?].

The discrete foam can be idealized as a compressible continuum when the length scales (associated with the geometry and loading) are much larger than the cell size [? ]. Size effects are exhibited when the structural length scale is comparable to the cell size (see e.g. [? ]), and the classical continuum theory must then be extended to include a characteristic length 
scale in the constitutive description, or the foam microstructure has to be explicitly modeled. Higher-order continuum theories have been used by? ] and ? ] to study the strain fields around a hole in a cellular solid. These theories were able to capture some of the observed cell size effects that were observed in the experiments of? ]. Discrete micromechanical models of a foam have also been used to study the strain fields around a sharp notch [? ? ]. Onck and co-workers [? ? ] have explored numerically the fracture of a discrete Voronoi structure under remote tension, and made from a strain-hardening solid. Each cell wall was modelled as an array of beam elements, and damage in the cell wall material was represented by a linear softening stress versus displacement response of the beam element beyond a critical value of stress. They found that the cell walls fail at random locations within the structure. Clusters of 2 to 3 failed struts eventually coalesce to form a single macroscopic crack which leads to final failure. Predictions have also been made for the case of regular hexagonal honeycombs in the elastic-brittle regime [? ] and in the creep ductile regime [? ] by assuming that each cell wall comprises several continuum elements in order to capture the scatter in the strut geometry (i.e. thickness and Plateau border radius). The failure of each strut was modelled by a linear softening constitutive response. The critical cluster size of the failed struts was found to be only a cell size for the elastic-brittle lattice and about half the width of the specimen for lattices in the creep ductile regime. These micromechanical models are able to explain the observed tensile ductility of foams, but their use is limited to small specimens containing only a few unit cells due to the associated computational cost.

In the present study, a cohesive zone approach is used to predict the fracture response of foams. The cohesive zone parameters representing the fracture process are deduced from the measured load versus displacement response of pre-cracked bend specimens. Predictions are then made for crack growth initiation and growth in metallic foams. The feasibility of the cohesive zone approach to model the fracture response is thereby assessed. We begin by reviewing the use of cohesive zone models in non-linear fracture mechanics.

\subsection{Cohesive zone models}

? ] considered crack growth at the tip of a long crack within an elasto-plastic solid of yield strength $\sigma_{\mathrm{Y}}$ and selected levels of strain hardening. The crack growth resistance curve (or 
$R$-curve) was predicted by placing a tensile cohesive zone of peak strength $\hat{\sigma}$ and of toughness $\Gamma_{0}$ (equal to the area under traction versus separation curve) along the crack plane. The initiation fracture toughness $K_{0}$ for the onset of crack growth follows directly from the Irwin relation $E \Gamma_{0}=\left(1-v^{2}\right) K_{0}^{2}$ in terms of the Young's modulus $E$ and Poisson ratio $v$ of the bulk solid. For the choice $\hat{\sigma} \leq 3 \sigma_{\mathrm{Y}}$, the cohesive zone strength $\hat{\sigma}$ is too small to activate plasticity within the surrounding bulk solid, resulting in a flat $R$-curve ${ }^{1}$. In contrast, a rising $R$-curve was observed for $\hat{\sigma}>3 \sigma_{\mathrm{Y}}$ with the elevation in crack growth resistance extremely sensitive to the value of $\hat{\sigma} / \sigma_{\mathrm{Y}}$. For example, for a solid with mild strain-hardening, the steady state value of fracture toughness $K_{\mathrm{SS}}$ (at large crack extensions) increases from $2.2 K_{0}$ when $\hat{\sigma}=3.5 \sigma_{\mathrm{Y}}$ to $4 K_{0}$ when $\hat{\sigma}=3.75 \sigma_{\mathrm{Y}}$. ? ] made similar predictions for the $R$-curve in an elastic-plastic compressible solid. They found that $K_{\mathrm{SS}} / K_{0}$ equals unity for $\hat{\sigma} \approx \sigma_{\mathrm{Y}}$ and increases dramatically with increasing $\hat{\sigma} / \sigma_{\mathrm{Y}}>1$. This reduction in critical value of $\hat{\sigma}$ from $3 \sigma_{\mathrm{Y}}$ to $\sigma_{\mathrm{Y}}$ is a direct consequence of the reduction in plastic constraint at the crack tip in a compressible solid. It is apparent from the studies of ? ] and of? ] that the solid must possess sufficient strain hardening in order to attain the prescribed magnitude of peak traction $\hat{\sigma}$, and for crack growth to ensue.

It is emphasised that the accuracy of the cohesive zone approach is sensitive to the specification of the cohesive traction versus separation law that describes the fracture process. In general, the cohesive law has 3 main features: (i) a peak value of traction, $\hat{\sigma}$, (ii) a softening curve associated with the tensile fracture process, and (iii) a toughness, $\Gamma_{0}$ (as defined by the area under the traction versus separation curve). Inverse analysis methods have been used extensively in the literature to determine the precise form of the cohesive law; see? ] for a review of these methods. They include a standard J-integral approach for a crack in a double cantilever beam specimen wherein the cohesive traction is computed as the derivative of the $J$-integral with respect to the crack tip opening displacement. However, it is challenging to deduce the cohesive zone law from a single geometry and then use it to predict the collapse response of a different geometry or loading condition.

\footnotetext{
${ }^{1}$ The factor of 3 closely relates to the Prandtl solution for peak traction ahead of the crack tip in an elastic perfectly-plastic incompressible solid.
} 


\subsection{Scope of study}

The aim of this study is to attempt to measure the toughness of an open-cell aluminium alloy foam, and to interpret the toughness in terms of the underlying microstructure. The J-integral test method, as specified in ASTM E1820, is adopted for single-edge-notch-bend (SENB) specimens. The direct current potential drop (DCPD) method is used to infer the degree of crack growth, and Digital Image Correlation (DIC) is used to measure the plastic zone size. The fracture process zone is observed by 3D X-ray computed tomographic (XCT) reconstructions of the specimen, at regular intervals during the test. Thus, the existence of a near tip J-field is investigated.

A cohesive zone, embedded in a compressible, elastic-plastic hardening solid, is used to predict the fracture response of the foam. The cohesive zone parameters, peak strength and toughness, are deduced from a goodness-of-fit measure between the measured and predicted load versus displacement response of the SENB specimens. The ability of the cohesive zone model to predict the fracture response of the foam is thereby explored.

\section{Experimental investigation}

\subsection{Material specification and experimental programme}

Flat panels of open-cell Al6101-T6 foam ${ }^{2}$, of dimension $300 \mathrm{~mm}$ x $300 \mathrm{~mm}$ x $26.4 \mathrm{~mm}$, were obtained in two (nominal) relative densities, $\bar{\rho}=6.6 \%$ and $\bar{\rho}=9.6 \%$. The foam microstructure was interrogated by X-ray computed tomography, see Figs. 2(a) and (b). For both relative densities of foam, the mean cell edge length is $\ell=1.96 \mathrm{~mm}$.

The foam panels were machined into the following 4 geometries: (i) dogbone-shaped specimen, as shown in Fig. 3(a), for measuring the tensile response of the foam, (ii) compression test specimen, as shown in Fig. 3(b), (iii) single edge notched bend (SENB) specimen for measuring toughness, see Fig. 3(c), and (iv) bend specimen without a pre-notch in order to assist in the validation of the foam constitutive model as used in the FE simulations. Local vari-

\footnotetext{
${ }^{2}$ Manufactured by ERG Aerospace Corporation, Oakland, California, USA. Refer to ? ] for details on the manufacturing process.
} 
ations in the foam microstructure are accompanied by a variation in the relative density $\bar{\rho}$ from specimen to specimen; consequently, the precise value of $\bar{\rho}$ for each test specimen was measured by weighing with a digital balance.

Uniaxial tension tests were performed on the dogbone-shaped specimens of Fig. 3(a) using a screw-driven test machine at a constant displacement rate of $0.01 \mathrm{~mm} / \mathrm{s}$. The crosssectional area of the uniform section was $30 \mathrm{~mm} \times 26.4 \mathrm{~mm}$, and the total length of the specimen (excluding the end tabs) was $100 \mathrm{~mm}$. The foaming rise direction of all specimens is the out-of-plane $z$-direction, see Fig. 3(a). The end tabs of the specimen were filled with epoxy for local reinforcement. Circular holes of diameter $10 \mathrm{~mm}$ were drilled at the centre of the epoxy-filled tabs to facilitate pin-loading of the specimen in the axial $y$-direction. Four repeat tests were performed for each value of $\bar{\rho}$. The load was measured by the load cell clamped to the stationary platen of the rig, and the average axial strain in the specimen was measured by an extensometer of gauge length $L_{g}=50 \mathrm{~mm}$ attached at mid-length of the specimen, see Fig. 3(a).

Uniaxial compression tests were performed on specimens of geometry shown in Fig. 3(b) to measure the in-plane response in the $x$ and $y$ directions. The shortening of the specimen was used to define the compressive strain in the specimen. Both platens were lubricated with PTFE spray to reduce friction. Four repeat tests for each value of $\bar{\rho}$ were performed at a constant displacement rate of $0.01 \mathrm{~mm} / \mathrm{s}$. The plastic Poisson's ratio $v^{\mathrm{P}}$ of the foam was defined as the negative ratio of the lateral logarithmic strain to the axial logarithmic strain. The value of $v^{\mathrm{P}}$ was determined from the uniaxial compression test using Digital Image Correlation (DIC) by tracking two facet points, one on each lateral edge of the specimen (with respect to the loading direction), during the course of the test.

\section{$2.2 J$-test procedure}

The crack growth resistance of the aluminium alloy foam was determined from the single edge notch bend (SENB) specimens, following the J-integral test procedure as outlined in ASTM standard E1820 [? ]. The direct current potential drop (DCPD) method was used to infer crack extension in the foam specimen. Details of the specimen geometry and test apparatus 
are given in Fig. 3(c). The test procedure is summarised below.

\subsubsection{Specimen size and loading}

The length of pre-crack, $a_{0}$, and of ligament, $W-a_{0}$, were chosen to satisfy the ASTM requirements for a valid $J_{\text {IC }}$ test (for fully dense metals) as given by:

$$
\left(a_{0}, W-a_{0}\right) \geq 25 \delta_{\mathrm{Tc}}=25 \frac{J_{\mathrm{Q}}}{\sigma_{\mathrm{Y}}}
$$

where $\delta_{\mathrm{Tc}}$ is the critical crack tip opening displacement, $J_{\mathrm{Q}}$ is the provisional value of initiation toughness from the single specimen technique prescribed in ? ], and $\sigma_{\mathrm{Y}}$ is the tensile yield strength of the foam as measured from the uniaxial tensile test. The size criterion of Eq. (1) demands that $\left(a_{0}, W-a_{0}\right)>9 \mathrm{~mm}$ for both $\bar{\rho}=6.6 \%$ and $\bar{\rho}=9.6 \%$. Accordingly, we chose $a_{0}=20 \mathrm{~mm}$ and $W=50 \mathrm{~mm}$ for all SENB specimens tested in this study. The specimens were of span $S=200 \mathrm{~mm}$ between the support rollers and of thickness $B=26.4 \mathrm{~mm}$. The pre-crack was machined using a fine blade of thickness $300 \mu \mathrm{m}$ which is much less than the cell size of $\ell=1.96 \mathrm{~mm}$.

Specimens were loaded in three-point bending via rollers of diameter $13 \mathrm{~mm}$, using a screwdriven testing machine at a constant displacement rate of $0.01 \mathrm{~mm} / \mathrm{s}$. These rollers ensured that the specimen was electrically insulated from the test machine to facilitate accurate potential drop measurements. The load $P$ was measured via a $2 \mathrm{kN}$ load cell clamped to the stationary platen of the rig. The crosshead displacement $v$ was tracked by a non-contact laser extensometer in addition to the DIC instrumentation. Prior to the start of the test, the specimens were spray-painted by black paint in order to enhance the speckle contrast of the DIC images. A single camera of the GOM system ${ }^{3}$ was used to track a facet of size $20 \times 20$ pixels on the foam directly beneath the point of contact with the loading roller. An array of additional facets were placed (by the DIC post-processing software) at suitable locations on the surface of the foam specimen to enable the measurement of crack mouth and crack tip opening displacements, and to determine the plastic zone size.

\footnotetext{
${ }^{3}$ GOM ARAMIS $12 \mathrm{M}$ system, maximum resolution: $4096 \times 3072$ pixels, $100 \mathrm{~mm}$ lens
} 


\subsubsection{Measurement of crack extension by direct current potential drop method}

A constant current of $1 \mathrm{~A}$ was applied between the ends of the specimen, see Fig. 3(c). The resulting voltage across the crack mouth was measured via soldered probe wires of diameter $0.2 \mathrm{~mm}$. The location of these probe wires is included in Fig. 3(c), and the voltage across the probe wires was recorded by a data logger. The relationship between the potential drop across probe wires and the crack length was obtained using the electrical analogue method as described by ? ]. Write $V_{\mathrm{o}}$ as the voltage across the probe wires for a normalised crack length $a / W$ equal to $a_{0} / W=0.4$ for the SENB specimen. Then, the relationship between the normalised crack length $a / W$ and the normalised voltage $V / V_{0}$ was obtained from regression fitting of the data from 3 repeat tests of the electrical analogue to give

$$
\frac{a}{W}=-0.01478+0.50116\left(\frac{V}{V_{\mathrm{o}}}\right)-0.0894\left(\frac{V}{V_{\mathrm{o}}}\right)^{2}+0.00557\left(\frac{V}{V_{\mathrm{o}}}\right)^{3}
$$

The voltage drop $V / V_{0}$ increases with increasing crack length $a / W$ according to Eq. (2). The increase in voltage (and resistivity) with distributed strut failure has been noted previously by ? ] for the same composition of aluminium alloy foam tested under uniaxial tension and without a pre-crack.

Unloading compliance techniques were used to verify the accuracy of the potential drop method in the toughness tests. The specimens were unloaded by $10 \%$ of the current load, and then re-loaded at regular intervals during the test. The DIC technique was used to track the crack mouth opening displacement and the load-line displacement during the toughness tests.

\subsection{Damage visualisation using X-ray computed tomography}

Three-dimensional computer-assisted X-ray tomography (XCT) was used to map the 3D fracture pattern of failed struts in the foam at selected stages of crack extension $\Delta a_{\mathrm{PD}}$, as inferred from the potential drop method. The toughness test was interrupted at selected values of $\Delta a_{\mathrm{PD}}$ and the crack was held open by wedging a PMMA sheet between the crack faces prior to unloading of the specimen in the test machine. High resolution 3D XCT scans of the specimen were obtained by placing the region of interest (in the vicinity of the crack tip) in the detector 
field-of-view; the typical voxel size of the scans was $30 \mu \mathrm{m}$. A series of post-processing steps were carried out in order to identify the broken struts and thereby compute the fracture process zone. The details are as follows. First, the 2-dimensional grey-scaled images from the XCT were stacked in a series of thin slices of height $\Delta W=1.5 \mathrm{~mm}$, length $L=20 \mathrm{~mm}$, and depth $B=26.4 \mathrm{~mm}$ into the page, as sketched in Fig. 3(d). Each slice was binarised to separate an intact aluminium strut (white) from air (black) by following the method as described in [? ]. The binarised images were cleaned by removing islands of single pixels or pixels with up to 4 neighbours. Next, the cleaned 3D image of each slice was projected onto a plane by assigning specific grey-scale value to each pixel depending upon its location in the slice. This technique enables the visual identification of the failed struts as well as their location. Finally, an image processing software ${ }^{4}$ was used to catalogue the number and the $(x, y, z)$ coordinates of the mid-point of each failed strut.

\section{Material characterisation}

The in-plane tensile and compressive responses of Al alloy foam of relative density $\bar{\rho}=6.6 \%$ and 9.6\% are shown in Fig. 4 in terms of the nominal stress $\sigma$ versus nominal strain $\varepsilon$. Data are shown for 4 nominally identical specimens for each relative density, with the solid line corresponding to the mean response and the shaded region representing the scatter in data. The initial response in both uniaxial tension and compression involves elastic bending of the cell walls. In uniaxial compression, yield occurs at a constant plateau stress prior to significant hardening at a nominal strain beyond $30 \%$. In contrast, under remote tensile loading, a few cell walls within a narrow band in the gauge section undergo tensile failure almost immediately after yield, and this leads to a softening post-yield response. The foam ductility is between $2 \%$ and $5 \%$ depending upon relative density, see Fig. 4 . The out-of-plane compressive strength was higher than the in-plane compressive strength by approximately $10 \%$ for both values of relative density. This mild anisotropy is attributed to the foaming process and is ignored in the remainder of the study. The ultimate tensile strength (UTS) is typically $10 \%$ below the in-plane compressive plateau stress $\sigma_{\mathrm{Y}}$ for both values of $\bar{\rho}$, as seen

\footnotetext{
${ }^{4}$ Fiji software, https://fiji.sc
} 
from Fig. 4.

The Young's modulus $E$ of each foam is measured from the slope of the unloading curve of the in-plane compressive response at a small value of plastic strain on the order of $1 \%$. Power-law fits to the measured mean values of $E$ and $\sigma_{\mathrm{Y}}$ provide scaling laws for $E$ and $\sigma_{\mathrm{Y}}$ in terms of the foam relative density $\bar{\rho}$ :

$$
\frac{E}{E_{\mathrm{S}}}=\bar{\rho}^{2.14} \text { and } \quad \frac{\sigma_{\mathrm{Y}}}{\sigma_{\mathrm{YS}}}=0.7 \bar{\rho}^{1.71}
$$

where $E_{\mathrm{S}}$ and $\sigma_{\mathrm{YS}}$ are the Young's modulus and yield strength, respectively, of the fully dense aluminium 6101 alloy. Here, $E_{\mathrm{S}}$ equals $70 \mathrm{GPa}$ and $\sigma_{\mathrm{YS}}$ equals $200 \mathrm{MPa}$, as taken from ? ]. The exponents on $\bar{\rho}$ in Eq. (3) are broadly consistent with bending of the cell walls of the foam: simple analytical models [? ] suggest $E \propto \bar{\rho}^{2}$ and $\sigma_{\mathrm{Y}} \propto \bar{\rho}^{3 / 2}$.

The plastic Poisson's ratio $v^{\mathrm{P}}$ of the foam was measured from the uniaxial compression tests using DIC software. The lateral strain in each specimen was measured at 5 equally spaced transverse sections of the specimen at a compressive strain of $10 \%$. The mean value of $v^{\mathrm{P}}$ from 4 nominally identical specimens for each value of $\bar{\rho}$ was found to be $v^{\mathrm{P}}=0.17$ for $\bar{\rho}=6.6 \%$ and $\nu^{\mathrm{P}}=0.25$ for $\bar{\rho}=9.6 \%$.

\section{Fracture tests}

\subsection{Crack growth resistance curves}

The response of the deep notched bend specimen is shown in Fig. 5 in terms of the load $P$ and crack extension $\triangle a_{\mathrm{PD}}$ from DCPD as a function of cross-head displacement $v$. Data are shown for 4 repeat tests for $\bar{\rho}=6.6 \%$ and $9.6 \%$, with the solid line corresponding to the mean response from the 4 tests and the shaded region representing the scatter in data. In all cases, crack growth (as inferred from the potential drop method) initiates prior to peak load. The accuracy of the potential drop method was verified by additionally measuring the crack extension by the unloading compliance method as described in? ]. Acceptable agreement was found between the unloading compliance and potential drop measurements.

The $J_{\mathrm{R}}$ versus $\Delta a_{\mathrm{PD}}$ crack growth resistance curves are obtained from the $P$ versus $v$ and $\Delta a_{\mathrm{PD}}$ 
versus $v$ curves following the steps outlined in ? ]; these responses are shown in Fig. 5(c) for both values of $\bar{\rho}$. Significant $R$-curve behaviour is observed for both values of relative density. The extent of the plastic zone ahead of the crack tip was measured using surface strain mapping by the DIC software. Contours of strain $\varepsilon_{y y}$ are shown in Fig. 5(d) at peak load for one specimen of $\bar{\rho}=6.6 \%$; it reveals the existence of a plastic zone on the order of the crack length at the onset of crack growth. The $R$-curve of the foam is attributed to two distinct zones of energy dissipation: (i) the bulk plastic zone emanating from the crack tip (containing a distribution of broken struts), and (ii) the crack bridging zone behind the advancing crack tip.

The value of toughness at crack initiation $J_{\mathrm{IC}}$ is determined from the $J-R$ resistance curves as follows. A crack blunting line, $J_{R}=2 \sigma_{\mathrm{Y}} \Delta a$, is drawn as shown in Fig. 5(c), and an offset line is drawn parallel to the blunting line, intersecting the abscissa at $0.2 \mathrm{~mm}$. The value of the initiation toughness $J_{\mathrm{IC}}$ is given by the intersection of the $J-R$ curve with the $0.2 \mathrm{~mm}$ offset line. Following this procedure, we obtain $J_{\text {IC }}=0.5 \mathrm{kNm}^{-1}$ for $\bar{\rho}=6.6 \%$ and $J_{\text {IC }}=0.85 \mathrm{kNm}^{-1}$ for $\bar{\rho}=9.6 \%$.

\subsection{Extent of the process zone due to strut failure}

A distribution of failed struts forms a fracture process zone at the crack tip. The location of all failed struts at a given value of crack extension was obtained by X-ray computed tomography, as described previously in Section 2.3. Consider one specimen of $\bar{\rho}=6.6 \%$ in detail. The distribution of failed struts projected over all $z$ in the $x-y$ plane, and projected over all $y$ in the $x-z$ plane is shown in Fig. 6(a) for $\Delta a_{\mathrm{PD}}=2 \mathrm{~mm}$ and in Fig. 6(b) for $\Delta a_{\mathrm{PD}}=10 \mathrm{~mm}$. We emphasise that the markers in the projected $x-y$ plane indicate the $(x, y)$ location of the mid-point of each failed strut over all values of $z$, that is $0 \leq z \leq B$. Likewise, the markers in the projected $x-z$ plane indicate the $(x, z)$ location of the mid-point of each failed strut over for all values of $y$ such that $-L / 2 \leq y \leq L / 2$. The damage parameter $f$ as a function of location $x$ at any value of $\Delta a_{\mathrm{PD}}$ is obtained as follows. We first identify the number of failed struts $n_{\mathrm{f}}$ within each control volume of $\Delta W L B$ (where $\Delta W=1.5 \mathrm{~mm}, L=10 \mathrm{~mm}$, and $B=26.4 \mathrm{~mm}$ ) corresponding to a traction-free fracture surface. The average value of $n_{\mathrm{f}}(x)$ is 22 , see for example Fig. $6(\mathrm{~b})$, for control volumes at $x<6 \mathrm{~mm}$. Second, we count the 
number of failed struts $n(x)$ within each control volume centred at $x$. Then, the fraction of failed struts $f(x)$ is defined as $f=n / n_{\mathrm{f}}$. The limit $f=1$ is a somewhat severe requirement due to scatter in material ductility, and scatter in the value of $n_{\mathrm{f}}(x)$ in the definition of a traction-free crack, and so we arbitrarily assume that $f=0.9$ corresponds to a traction-free crack extension (i.e. no bridging ligaments) whereas $f=0$ corresponds to a region where no struts have failed. The distribution of failed struts ahead of the initial crack tip is shown in Fig. 6(c) for the choice $\Delta a_{\mathrm{PD}}=10 \mathrm{~mm}$. The region over which $0 \leq f \leq 0.9$ can be interpreted as a fracture process zone (FPZ), or equivalently a crack bridging zone (depending upon the assumed location of the crack tip). For definiteness, we shall assume that the physical crack tip exists at the transition point from $f \equiv 0$ to $f>0$. Define the maximum extent of the FPZ, $\Delta a_{\mathrm{D}}$, as the distance along the $x$-direction from the initial crack tip to the nearest location of $f=0$. The traction-free crack extension $\Delta a_{\mathrm{f}}$ is the length over which $f \geq 0.9$ ahead of the initial crack tip. We note from Fig. 6(c) that an inferred crack extension of $\Delta a_{\mathrm{PD}}=10 \mathrm{~mm}$ corresponds to traction-free extension of $\Delta a_{\mathrm{f}}=8.4 \mathrm{~mm}$ and a FPZ of length $\Delta a_{\mathrm{D}}=18.3 \mathrm{~mm}$ ahead of the current location of the traction-free crack tip.

The extent of damage $f$ is plotted as a function of location $x$ ahead of the initial crack tip in Fig. 7(a) for selected values of $\Delta a_{\mathrm{PD}}$ in the range $1 \mathrm{~mm}$ to $10 \mathrm{~mm}$. The relation between $\Delta a_{\mathrm{f}}$, $\Delta a_{\mathrm{D}}$, and $\Delta a_{\mathrm{PD}}$ is given in Fig. 7(b). Note that, when $\Delta a_{\mathrm{PD}}=2 \mathrm{~mm}$, the FPZ is of size $\Delta a_{\mathrm{D}}=$ $7.4 \mathrm{~mm}$ and the traction-free crack extension $\Delta a_{\mathrm{f}}$ vanishes. In general, the traction-free crack tip lags behind the inferred crack tip from PD measurements by about $4 \mathrm{~mm}$. Further, the extent of the bridging zone $\Delta a_{\mathrm{D}}$ increases steeply with crack extension: $\Delta a_{\mathrm{D}}=3.4 \mathrm{~mm}$ at $\Delta a_{\mathrm{PD}}=1 \mathrm{~mm}$ and $\Delta a_{\mathrm{D}}=18.3 \mathrm{~mm}$ at $\Delta a_{\mathrm{PD}}=10 \mathrm{~mm}$. It is instructive to compare the relative extent of crack tip plastic zone $r_{\mathrm{P}}$ and the FPZ $\Delta a_{\mathrm{D}}$ in order to assess whether a crack tip Jfield exists. As an approximation, assume that the foam yields when the von Mises measure of strain exceeds a value of $\sigma_{\mathrm{Y}} / E$, and define $r_{\mathrm{P}}$ as the maximum extent of plastic zone from the crack tip. The von Mises strain $\varepsilon_{\mathrm{e}}$ was calculated from the measured values of minor principal strain $\varepsilon_{1}$ and the major principal strain $\varepsilon_{2}$ on the $z=0$ plane as

$$
\varepsilon_{\mathrm{e}}^{2}=\frac{2}{9}\left[\left(\varepsilon_{1}-\varepsilon_{2}\right)^{2}+\varepsilon_{1}^{2}+\varepsilon_{2}^{2}\right]
$$


assuming plane strain, $\varepsilon_{3} \equiv 0$. The values of $\varepsilon_{1}$ and $\varepsilon_{2}$ were obtained from the DIC software based on facets of size on the order of the foam cell size. The extent of plastic zone $r_{\mathrm{P}}$ is plotted in Fig. 7(b) as a function of crack extension for $\bar{\rho}=6.6 \%$. Plots similar to Figs. 7(a) and (b) have also been generated for the case of $\bar{\rho}=9.6 \%$, see Figs. $7(\mathrm{c})$ and (d).

We conclude from Figs. $7(\mathrm{~b})$ and $(\mathrm{d})$ that the FPZ size $\Delta a_{\mathrm{D}}$ in the Al alloy foams is on the order of the plastic zone size at the onset of traction-free crack extension, and for subsequent crack growth. Consequently, a crack tip J-field does not exist, and the measured value of $J_{\text {IC }}$ following the ASTM $J$-integral test procedure cannot be treated as a material property despite the specimen size meeting the criteria of the ASTM standard. We emphasise that the ASTM procedure is based on the assumption that the FPZ is much smaller than the plastic zone size. This criterion is obeyed in fully dense metals but is violated for the metal foam under current consideration. Note that the fracture toughness $K_{\mathrm{IC}}$ remains a valid material parameter for the foam provided the specimen size is sufficiently large for an outer $K$-field to exist. Recall that the ASTM guideline suggests the following dimensions for a valid $K_{\mathrm{IC}}$ test on a single edge notch bend geometry:

$$
\left(a_{0}, W-a_{0}, B\right)>2.5\left(\frac{K_{\mathrm{IC}}}{\sigma_{\mathrm{Y}}}\right)^{2}
$$

This size criterion remains meaningful, but we cannot estimate the value of $K_{\mathrm{IC}}$ from the value of $J_{\text {IC }}$ from a $J$-test. We emphasise that this case of metals foams is different from situations such as those discussed by ? ] where $J$ is an insufficient parameter to characterise fracture in fully dense metals. In those cases a $J$-field exists but fracture is dependent on both the J-field and the so-called non-singular Q term, introduced by O'Dowd and Shih [? ? ].

\subsection{Conditions for the existence of a crack tip $J$-field: fully dense alloys versus micro- architected materials}

Recall that the criterion for the existence of a crack tip J-field in a fully dense alloy is given by Eq. (1), consistent with Fig. 1(a). Additional length scales arise in metallic foams (and in lattice materials in general): the cell size $\ell$ and strut thickness $t$. The criterion for the 
existence of a crack tip J-field may thus differ from that stated in Eq. (1). A J-field can only exist if the plastic zone spans a minimum number $n_{1}$, of cells, such that $r_{\mathrm{P}}>n_{1} \ell$ (where the precise value of $n_{1}$ requires future study). Further, the FPZ must be contained within the zone of $J$-dominance. This criterion is achievable when fracture occurs in a sequential fashion strut-by-strut at the crack tip. In contrast, when struts fail stochastically in a zone of size comparable to that of the plastic zone, an annular zone of $J$-dominance does not surround the FPZ, recall Fig. 1(b). In this case, no near-tip J-field exists and $J$ cannot be used as a fracture parameter. This is the case for the current metallic foam under consideration.

\section{Fracture model}

Can a cohesive zone model be used to predict the fracture response of the foam of the present study? To address this, the cohesive zone method of? ] is used to model crack advance in the deep-notched bend (SENB) specimen. This method allows for crack bridging in the presence of bulk plasticity as observed in the metal foam without assuming a priori that the bridging zone is much smaller than the plastic zone. Static finite element (FE) calculations were performed with ABAQUS/Standard v6.14 to aid interpretation of the experimental observations such as the $R$-curve and the bridging zone. The foam is modelled as an isotropic, compressible elastic-plastic strain-hardening solid, based on the compressible elastic-plastic constitutive model of ? ]. The objectives of the numerical study are: (i) to deduce the cohesive parameters such as the peak traction $\hat{\sigma}$ and toughness $\Gamma_{0}$ based on the measured load versus displacement response, and (ii) to determine whether the cohesive zone model can predict the extent of crack growth.

\subsection{FE model}

The FE mesh assumes that the notch in the SENB specimen has a semi-circular tip ${ }^{5}$ of diameter $d=\ell$, where $\ell=2 \mathrm{~mm}$ is the average cell edge length of the foam. Introduce the co-ordinate system $(x, y)$ as shown in Fig. 8. A cohesive zone is placed ahead of notch on $a_{0} \leq x \leq W$ comprising four-noded cohesive elements (type COH2D4 in ABAQUS) of zero

\footnotetext{
${ }^{5} \mathrm{~A}$ series of additional FE simulations were performed with a sharp crack tip. It was found that the notch acuity has a negligible effect upon the load versus displacement response.
} 
thickness. The FE mesh for the foam comprises linear quadrilateral elements of plane strain (type CPE4R). All rollers are modelled as rigid surfaces, and frictionless contact is assumed between the rollers and foam. A symmetric half model is employed in the FE study with the support roller fixed in all directions and the loading roller subjected to a constant downward velocity in the $z$-direction. The velocity of loading is chosen to be sufficiently small for inertial effects to be negligible; the response obtained from the explicit FE simulation is thus quasi-static. Salient features of the assumed material models for the foam and the cohesive zone are outlined below.

\subsection{Material model for the foam}

The initial modulus of the foam (for $\bar{\rho}=6.6 \%$ ) is taken to be $E=175 \mathrm{MPa}$, based on the measured mean value of the unloading modulus during uniaxial compression test. An elastic Poisson's ratio of 0.3 is assumed, based on [? ]. The post-yield behaviour of the foam is modelled using the ABAQUS crushable foam model with isotropic hardening, based on the constitutive model of ? ], as follows. The yield surface is assumed to be elliptical, with the centre of the ellipse located at the origin of the mean stress versus von Mises effective stress plane. It evolves in a geometrically self-similar manner, and is of the form

$$
\phi=\hat{\sigma}-\sigma_{Y}=0
$$

where $\sigma_{\mathrm{Y}}$ is the uniaxial yield strength of the foam (assumed to be identical in tension and compression), and the effective stress $\hat{\sigma}$ is defined as

$$
\hat{\sigma}^{2}=\frac{1}{1+\left(\frac{\alpha}{3}\right)^{2}}\left(\sigma_{\mathrm{e}}^{2}+\alpha^{2} \sigma_{\mathrm{m}}^{2}\right)
$$

Here, $\sigma_{\mathrm{e}}$ is the von Mises effective stress, $\sigma_{\mathrm{m}}$ is the mean stress, and $\alpha$ is the shape factor of the yield ellipse. Associated plastic flow rule is assumed. Consequently, $\alpha$ is related to the plastic Poisson's ratio $v^{\mathrm{P}}$ of the foam according to

$$
\alpha^{2}=\frac{9}{2}\left(\frac{1-2 v^{\mathrm{P}}}{1+v^{\mathrm{P}}}\right)
$$


During plastic flow, the yield surface grows in a geometrically self-similar manner with strain, in accordance with the specified hardening response and the fixed value of shape factor $\alpha$ of the yield surface.

The initial yield strength $\sigma_{\mathrm{Y}}=1.42 \mathrm{MPa}$ of the foam (for $\bar{\rho}=6.6 \%$ ) is based on the measured mean value from 4 uniaxial compression tests, recall Fig. 4 . The assumed (idealised) true stress $\sigma$ versus true strain $\varepsilon$ response for the foam is shown in Fig. 9(a); it is derived from the mean of the measured nominal compressive stress $\sigma_{\mathrm{n}}$ versus nominal compressive strain $\varepsilon_{\mathrm{n}}$ responses of Fig. 4 using the following relation to account for plastic compressibilty:

$$
\sigma=\sigma_{\mathrm{n}}\left[\frac{1+\varepsilon_{\mathrm{n}}}{1+\left(1-2 v^{\mathrm{P}}\right) \varepsilon_{\mathrm{n}}}\right]
$$

A value of $v^{\mathrm{P}}=0.17$ is assumed for the foam of $\bar{\rho}=6.6 \%$ based on the measured mean value of $v^{\mathrm{P}}$ from 4 tests, recall Section 3. The shape factor $\alpha$ of the yield surface follows from Eq. (8) as $\alpha=1.58$.

\subsection{Cohesive zone model}

Crack advance from the tip of the pre-notch is modelled via the tensile traction versus opening displacement relation. It is assumed that crack growth occurs on the symmetry plane $y=0$. Consequently, it suffices to specify a relation for the normal traction $T$ and crack opening $\delta$. We adopt the XuâĂşNeedleman interfacial law [? ] for each cohesive element in both tension and compression; it has the form

$$
\frac{T}{\hat{\sigma}}=\frac{\delta}{\delta_{\mathrm{n}}} \exp \left(1-\frac{\delta}{\delta_{\mathrm{n}}}\right)
$$

where $\hat{\sigma}$ is the peak crack opening traction that occurs at an opening $\delta=\delta_{\mathrm{n}}$, as shown in Fig. 10(a). The fracture energy (or toughness) of the cohesive zone is the area under the $T$ versus $\delta$ curve: $\Gamma_{0}=\int_{0}^{\infty} T d \delta=e \hat{\sigma} \delta_{\mathrm{n}}$. The crack opening displacement $\delta$ is related directly to the material displacement along the centre-line of the specimen such that $\delta=2 u_{y}\left(0^{+}, x\right)=$ $-2 u_{y}\left(0^{-}, x\right)$.

The precise values of the cohesive properties $\left(\hat{\sigma}, \Gamma_{0}\right)$ for the foam are not known a priori; in- 
stead, we deduce values for $\left(\hat{\sigma}, \Gamma_{0}\right)$ based on a goodness-of-fit between the predicted and measured load versus displacement response during crack growth. To achieve this, a series of FE simulations were performed for selected values of $\hat{\sigma}$ from 1.42 $\mathrm{MPa}$ (equal to the initial yield strength of the solid $\sigma_{\mathrm{Y}}$ ) to $2 \mathrm{MPa}$, and $\Gamma_{0}$ in the range of $0.1 \mathrm{kNm}^{-1}$ to $1 \mathrm{kNm}^{-1}$. Recall that the characteristic cohesive length $\ell_{c}$ is defined by

$$
\ell_{\mathrm{c}}=\frac{\pi}{8} \frac{E \Gamma_{0}}{\hat{\sigma}^{2}}
$$

In order to ensure adequate mesh resolution during all stages of crack growth, $0 \leq \Delta a \leq$ $W-a_{0}$, a uniform FE mesh of element size $\ell_{\mathrm{e}}$ (for both the solid and the cohesive zone) is constructed across the ligament such that $\ell_{\mathrm{e}} \leq 0.05 \ell_{\mathrm{c}}$, see Fig. 8 .

\section{Predicted fracture response}

\subsection{Validation of the foam constitutive model}

In order to verify the accuracy of the foam constitutive model, an FE simulation is performed for the case of 3-point bending of the foam specimen absent a pre-notch (and without a cohesive zone). The relevant dimensions of this specimen are: $S=200 \mathrm{~mm}, W=30 \mathrm{~mm}$, and plane strain thickness $B=26.4 \mathrm{~mm}$.

The measured mean curve of the load versus displacement response is shown in Fig. 9(b); the load $P$ is normalised by the plastic collapse load, $\sigma_{\mathrm{Y}} B W^{2} / S$, and the roller displacement $v$ is normalised by the span $S$. The peak load of the bend specimen is dictated by plastic collapse of the ligament of the specimen. Subsequently, tensile failure of the cell walls occurs at the outermost layer, and a crack propagates through the specimen, leading to a softening $P$ versus $v$ response. The FE prediction of the bend response is included in Fig. 9(b). Good agreement is noted between the FE prediction and the experiment until plastic collapse of the section at the mid-length $(y=0)$ occurs at $P S / \sigma_{\mathrm{Y}} B W^{2}=1$. Failure of the foam was not included in the FE model; the continued mild hardening in $P$ versus $v$ response for $v / S>0.03$ is a consequence of the assumed strain-hardening response of the foam. 


\subsection{Response of a deep notch specimen under 3-point bending: limiting cases}

Consider the following 3 limiting cases of cohesive zone properties: (i) $\hat{\sigma}=\Gamma_{0}=\infty$ such that softening and crack extension do not occur, (ii) a rigid, ideally plastic cohesive zone with $\hat{\sigma}=\sigma_{\mathrm{Y}}$ and $\Gamma_{0}=\infty$, and (iii) $\hat{\sigma}=\sigma_{\mathrm{Y}}$ and $\Gamma_{0}=0.1 \mathrm{kNm}^{-1}$ to simulate brittle fracture. (Note that the choice $\Gamma_{0}=0$ would require $\ell_{\mathrm{e}}=0$ in order to adequately resolve the crack tip field within the cohesive elements.) Denote the initial ligament length by $b_{0}$ such that $b_{0}=W-a_{0}$. The normalised load $P S / \sigma_{\mathrm{Y}} B b_{0}^{2}$ versus normalised displacement $v / S$ for these limiting cases are plotted in Fig. 10(b) and they demonstrate the bounds of the $P$ versus $v$ response that can be obtained from the cohesive zone model. The measured mean response lies within these bounds. The responses of cases (i) and (ii) are nearly identical due to the bulk plasticity at the notch tip and beneath the roller. The mild increase in load beyond the plastic collapse $\operatorname{load}\left(P S / \sigma_{\mathrm{Y}} B b_{0}^{2}=1\right)$ is due to the strain-hardening characteristic of the foam.

\subsection{Extraction of the cohesive parameters}

A series of FE simulations were performed using selected combinations of $\left(\hat{\sigma}, \Gamma_{0}\right)$, with $\hat{\sigma}$ between 1.42 $\mathrm{MPa}$ and $2 \mathrm{MPa}$, and $\Gamma_{0}$ between $0.1 \mathrm{kNm}^{-1}$ and $1 \mathrm{kNm}^{-1}$. Each FE simulation, obtained for a given combination of $\left(\hat{\sigma}, \Gamma_{0}\right)$, gives rise to the following quantities which can be compared with the experimental observations:

(i) load $P$ versus roller (or cross-head) displacement $v$ response,

(ii) traction-free crack extension $\Delta a_{\mathrm{f}}$ versus $v$,

(iii) evolution of the crack tip opening displacement $\delta_{\mathrm{T}}$ and crack mouth opening displacement $\delta_{\mathrm{M}}$ with increasing $v$,

(iv) FPZ size $\Delta a_{\mathrm{D}}$ (comprising cell wall failure and crack bridging), plastic zone size $r_{\mathrm{P}}$, and traction-free crack extension $\Delta a_{\mathrm{f}}$ ahead of the pre-notch tip, each versus $v$.

The crack tip opening displacement $\delta_{\mathrm{T}}$ and crack mouth opening displacement $\delta_{\mathrm{M}}$ are defined in Fig. 8: $\delta_{\mathrm{T}}$ is the change in distance between points T and T' placed on the diametric ends of the semi-circular notch tip, and $\delta_{M}$ is the change in distance between points $M$ and $M^{\prime}$ at the notch mouth. The precise locations of T, T', M, and $\mathrm{M}^{\prime}$ are identified (within $\pm 0.1 \mathrm{~mm}$ ) 
and tracked during the experiment using DIC software. Traction-free crack extension is assumed to occur in the FE simulation when the traction $T$ at an integration point within the cohesive element drops to $0.01 \hat{\sigma}$. The traction-free crack extension $\Delta a_{\mathrm{f}}$ in the experiments is determined from the 3D reconstruction of the fracture process zone based on a set of XCT scans, recall Fig. 7(a).

In order to extract the cohesive parameters $\left(\hat{\sigma}, \Gamma_{0}\right)$ associated with the fracture process, we define a goodness-of-fit measure, $\chi$, for the $P$ versus $v$ response as follows:

$$
\chi=\frac{\int_{0}^{v_{\mathrm{f}}}\left|P_{\exp }(v)-P_{\mathrm{FE}}(v)\right| d v}{\int_{0}^{v_{\mathrm{f}}} P_{\exp }(v) d v}
$$

Here, $P_{\exp }(v)$ is the load versus displacement response from the experiment; a mean response from 4 tests is assumed for the calculation of $\chi . P_{\mathrm{FE}}(v)$ is the predicted load versus displacement $v$ response and $v_{\mathrm{f}}$ is the maximum value of cross-head displacement $v$ in the experiment. A value of $\chi=1$ indicates perfect agreement between predicted and measured responses. Contours of $\chi$, as obtained from a set of $64 \mathrm{FE}$ simulations, are plotted in Fig. 11(a) with axes of cohesive strength $\hat{\sigma}$ and toughness $\Gamma_{0}$.

Three distinct local maxima of best fit ( $\chi \geq 0.9)$ emerge in the map of Fig. 11(a). We direct our attention towards the optimal point within each of these regions: point $\mathrm{A}$ with $\left(\chi, \hat{\sigma}, \Gamma_{0}\right)=$ $\left(0.91,1.47 \mathrm{MPa}, 0.96 \mathrm{kNm}^{-1}\right)$, point B with $\left(\chi, \hat{\sigma}, \Gamma_{0}\right)=\left(0.94,1.63 \mathrm{MPa}, 0.45 \mathrm{kNm}^{-1}\right)$, and point $\mathrm{C}$ with $\left(\chi, \hat{\sigma}, \Gamma_{0}\right)=\left(0.97,2.00 \mathrm{MPa}, 0.10 \mathrm{kNm}^{-1}\right)$. The corresponding cohesive laws for the 3 cases are shown in Fig. 11(b). The predicted load versus displacement response for cases A, B, and $\mathrm{C}$ are compared in Fig. 12(a) with the measured response. Note that the Dugdale plastic zone length as given by Eq. (11) is of magnitude $\ell_{\mathrm{c}}=30.5 \mathrm{~mm}, 11.7 \mathrm{~mm}$ and $1.7 \mathrm{~mm}$ for cases $A, B$ and $C$ respectively.

The crack tip opening displacement $\delta_{\mathrm{T}}$ and crack mouth opening displacement $\delta_{\mathrm{M}}$ for the three best fitting cases $\mathrm{A}, \mathrm{B}$, and $\mathrm{C}$ are almost indistinguishable, and they agree well with the corresponding measured data from DIC, see Fig. 12(b). Thus, it is not possible to distinguish the best choice of $\left(\hat{\sigma}, \Gamma_{0}\right)$ values on the basis of $P$ versus $v$ response alone. In order to gain further insight into the best choice of $\mathrm{A}, \mathrm{B}$, or $\mathrm{C}$, it is necessary to explore the accuracy of the 
predictions with additionally available experimental data, as follows.

Consider the cohesive law of Fig. 10(a). We assume that damage in the cohesive element initiates when the traction $T$ attains the peak value of $\hat{\sigma}$; the damage parameter $f$ at this instant is equal to zero. With increasing opening displacement $\delta, f$ increases until it becomes unity when $T$ drops to $0.01 \hat{\sigma}$, marking the onset of traction-free crack extension $\Delta a_{\mathrm{f}}$. The region over which $0 \leq f<0.9$ in the cohesive elements ahead of the pre-crack can be interpreted as the fracture process zone (FPZ), recall Section 4.2. Consequently, the maximum extent of the FPZ, $\triangle a_{\mathrm{D}}$, corresponds to the distance along the ligament from the initial crack tip to the location of $f=0$. Likewise, the traction-free crack extension $\Delta a_{\mathrm{f}}$ is the distance along the ligament from the initial crack tip over which $f$ attains or exceeds 0.9. The extent of damage zone $\Delta a_{\mathrm{D}}$ and the traction-free crack extension $\Delta a_{\mathrm{f}}$ from the FE simulations are shown in Figs. 12(c) and (d), respectively, as a function of roller displacement $v$, for the 3 best fitting cohesive laws. The measured values of $\Delta a_{\mathrm{D}}$ and $\Delta a_{\mathrm{f}}$, as taken from Fig. $5(\mathrm{~b})$ and Fig. 7(b), are included in Figs. 12(c) and (d), respectively. The ordinate in each case is normalised by the initial ligament length, $b_{0}=W-a_{0}$. We note from Figs. 12(c) and (d) that case A best predicts the evolution of damage zone $\Delta a_{\mathrm{D}}$ but case B provides best agreement with the observed traction-free crack extension $\Delta a_{\mathrm{f}}$. The cross-plot of $\Delta a_{\mathrm{f}}$ and $\Delta a_{\mathrm{D}}$ in Fig. 12(e) further demonstrates the large variation in response for cases A to C.

It is instructive to compare the extent of the plastic zone $r_{\mathrm{P}}$ at the tip of the pre-crack to the size of the FPZ. Predictions of $r_{\mathrm{P}}$ based on Eq. (4) are shown in Fig. 12(f) for the 3 best fitting cohesive laws along with the DIC measurement of $r_{\mathrm{P}}$. The FPZ size $\Delta a_{\mathrm{D}}$ is generally smaller than the plastic zone size $r_{\mathrm{P}}$, with the observed response sandwiched between the predictions for cases A and B. Further, we find from Figs. 12(e) and (f) that the initiation of crack growth $\left(\Delta a_{\mathrm{f}}=0^{+}\right)$is accompanied by a large FPZ in all 3 predictions (as well as in the experiment): $\Delta a_{\mathrm{D}} / r_{\mathrm{P}}=0.92$ for case $\mathrm{A}, \Delta a_{\mathrm{D}} / r_{\mathrm{P}}=0.36$ for case $\mathrm{B}$, and $\Delta a_{\mathrm{D}} / r_{\mathrm{P}}=0.1$ for case $\mathrm{C}$, at $\Delta a_{\mathrm{f}}=0^{+}$. These points are marked by the symbol O in Fig. 12(f) for clarity.

We conclude from Fig. 12 that no unique pair of $\left(\hat{\sigma}, \Gamma_{0}\right)$ examined here can simultaneously capture all the experimental observations: load, crack extension, and the development of the damage/bridging zone. The predictions of crack growth are sensitive to the choice of 
$\hat{\sigma} / \sigma_{\mathrm{Y}}$ : a value of $\hat{\sigma}$ less than $\sigma_{\mathrm{Y}}$ implies that the fracture process zone is embedded within an elastic solid, thereby leading to a flat $R$-curve. In contrast, a value of $\hat{\sigma}$ greater than $\sigma_{\mathrm{Y}}$ will give an increasing $R$-curve but the low strain hardening characteristic of the foam leads to an extreme sensitivity of the predicted response to the precise choice of $\hat{\sigma} / \sigma_{\mathrm{Y}}$. Additionally, we find that the values of $\hat{\sigma} / \sigma_{Y}$ that give best alignment between predicted and measured load versus displacement (as well as crack mouth and crack tip opening displacements) corresponds to tensile strains on the order of $40 \%-50 \%$ at the notch-tip; this is unrealistic for metal foams since their tensile ductility is on the order of a few percent $(2 \%-5 \%$, depending upon the relative density).

\section{An attempt to measure directly the cohesive zone law}

We proceed to explore whether the mode I cohesive law can be measured directly from the response of a deep-notch tensile specimen. In particular, the relationship between the cohesive zone law and the average traction versus additional axial displacement of a deeply notched specimen, is now determined. Here, we interpret the additional displacement as the additional elongation associated with the presence of the edge cracks and plasticity in the net section.

Double edge notch specimens of length $2 L=100 \mathrm{~mm}$, width $2 W=50 \mathrm{~mm}$, and thickness $B=26.4 \mathrm{~mm}$ were machined from a flat panel of $\bar{\rho}=6.6 \%$ foam. Notches of length $a$ were machined using a fine blade of thickness $300 \mu \mathrm{m}$, on both sides of the specimen, see Fig. 13(a). Two notch sizes were considered: $a=15 \mathrm{~mm}$ and $a=20 \mathrm{~mm}$. The extra displacement $\Delta u$ associated with plasticity and distributed cracking of the net section between the notches was measured using DIC by tracking two facet points spaced $10 \mathrm{~mm}$ apart and symmetrically about the mid-plane as shown in Fig. 13(a).

The load versus displacement response of the two deep notched specimens are shown in Fig. 13(b). The net section stress $\sigma^{\infty}$ in both cases is normalised by the un-notched compressive yield strength $\sigma_{\mathrm{Y}}$ of the foam, and is plotted against the gauge displacement $\Delta u$. The peak value of $\sigma^{\infty}$ for both the notch geometries slightly exceeds the yield strength of the foam, consistent with the notch strengthening behaviour observed in open-cell metallic 
foams due to size effects, see for example, ? ] and ? ].

Contours of the displacement $u_{y}$ in the loading direction at peak load, as obtained from the DIC, are shown in Fig. 14(a) and (b), for $a=15 \mathrm{~mm}$ and $a=20 \mathrm{~mm}$, respectively. Note that there is no significant displacement jump within a thin ligament between the notch tips. Consequently, the measured $\sigma^{\infty}$ versus $\Delta u$ response is not a direct measure of the crack bridging law. The DIC contours show a displacement jump $\Delta u_{y}$ of $0.4 \mathrm{~mm}$ across the gauge length of $10 \mathrm{~mm}$ at peak load: the observed failure strain of $4 \%$ from the DIC is consistent with the tensile ductility of the foam.

\subsection{An independent test that makes use of the calibrated cohesive law}

FE simulations were performed on the two double edge notch specimens $(a / W=0.6$ and $a / W=0.8$ ) by using the best calibration, case $B$, from the bend tests for the cohesive zone parameters. The $T$ versus $\delta$ curve for case B is included in Fig. 14(b); it differs from the measured $\sigma^{\infty}$ versus $\Delta u$ response in the softening portion of the curve. Predictions of the load versus displacement response are shown in Fig. 13(b) along with contours of displacement $u_{y}$ in Fig. 14(a) and (b). We find that the cohesive zone model gives an acceptable agreement of the load versus displacement response for the two deep notch tensile specimens with their corresponding measured response. However, in a similar manner to the bend test, the FE simulation predicts unrealistically high notch root strains (> 20\%) within a zone on the order of the cell size, see Fig. 14(c) for contours of strain at peak load.

\section{Concluding remarks}

The J-test procedure, as outlined in the ASTM standard E1820, is valid only when the fracture process zone is much smaller than the plastic zone surrounding the crack tip. This is not the case for the aluminium alloy foams of the present study. A crack tip $J$-field does not exist for the foams studied here, at any stage of crack growth. This is traced to the fact that a large fracture process zone exists ahead of the crack tip, and extends to almost the plastic zone boundary. The measured value of $J_{\text {IC }}$ is therefore not a material property. The present experimental study does not consider specimens that are sufficiently large to satisfy the ASTM 
criterion for a remote $K$-field to exist, recall Eq. (5). If tests were performed on specimens of sufficient size that an outer $K$-field exists, then this test could be used to measure $K_{\mathrm{IC}}$. Thus, $K_{\mathrm{IC}}$ remains a material property for the foam.

A cohesive zone model has the ability to capture the large scale bridging that occurs in metal foams, but it remains a challenge for the model to capture the main features of crack advance that are observed in the experiment in addition to the load versus displacement collapse response. In the present study, the cohesive zone model of ? ], along with a compressible plasticity model for the foam, was used to model crack growth in a deep notched bend specimen, and thereby used to extract the cohesive zone parameters: the cohesive strength and toughness. Three possible combinations of cohesive strength and toughness give an acceptable agreement with the measured load versus displacement response. However, the value of $\hat{\sigma} / \sigma_{\mathrm{Y}}$ that gives best agreement with the measured load versus displacement (as well as crack mouth and crack tip opening displacements) demands the existence of tensile strains on the order of $40 \%-50 \%$ at the notch-tip. This is unrealistic since the foam has a tensile ductility of only a few percent. None of the 3 choices of cohesive zone law was able to predict accurately the degree of crack extension (bridged crack and traction-free crack) as a function of remote displacement.

\section{Acknowledgements}

The authors gratefully acknowledge financial support from the European Research Council (ERC) in the form of advanced grant, MULTILAT, GA669764. We are also grateful for enlightening discussions with Prof. John W. Hutchinson on the topic of cohesive zone modelling. 


\section{List of Figures}

1 (a) A fracture process zone (FPZ) embedded within a crack tip J-field; (b) no crack tip J-field is present due to the existence of a FPZ comparable in dimension to the plastic zone size. . . . . . . . . . . . . .

2 X-ray CT images of aluminium alloy foam of (a) relative density $\bar{\rho}=6.6 \%$ and (b) $\bar{\rho}=9.6 \%$. The scale bar is of length $1 \mathrm{~mm} . \ldots \ldots \ldots$

3 Specimen geometries for (a) uniaxial tension test, (b) uniaxial compression test, and (c) fracture toughness test along with the apparatus. The out-ofplane thickness of all specimens is $B=26.4 \mathrm{~mm}$. (d) Definition of a slice used in the XCT analysis for damage visualisation. . . . . . . . . . . .

4 Uniaxial tension, and in-plane compression, stress versus strain curves for the

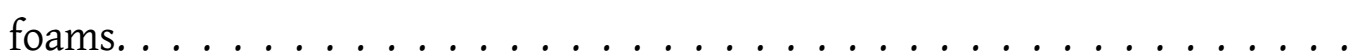

$5 \quad$ Fracture response of deep-notched bend specimens: (a) Load $P$ versus crosshead displacement $v$ response, (b) crack extension $\Delta a_{\mathrm{PD}}$, as measured from the DCPD method, versus $v$, (c) crack growth resistance curves for $\bar{\rho}=6.6 \%$ and $9.6 \%$ specimens, and (d) DIC contours of longitudinal strain $\varepsilon_{y y}$ in a specimen of $\bar{\rho}=6.6 \%$ at peak load. The yield strain of this foam is $\varepsilon_{\mathrm{Y}}=\sigma_{\mathrm{Y}} / E=0.008 .31$

6 Distribution of failed struts as determined from XCT analysis in a representative specimen of $\bar{\rho}=6.6 \%$ : (a) Location of failed struts for $\Delta a_{\mathrm{PD}}=2 \mathrm{~mm}$ : the projected view in the $(x, y)$ plane shows failed struts over all $z$; likewise, the view in the $(x, z)$ plane shows failed struts over all $y$-values. (b) Location of failed struts for $\Delta a_{\mathrm{PD}}=10 \mathrm{~mm}$ : again, the projected view in the $(x, y)$ is over all $z$; likewise, the view in the $(x, z)$ plane is over all $y$. (c) fraction of failed struts $f$ along the ligament $\left(x\right.$-direction) for $\Delta a_{\mathrm{PD}}=10 \mathrm{~mm} . \ldots \ldots$ 
$7 \quad$ Extent of crack bridging for $\bar{\rho}=6.6 \%$ : (a) $f$ versus distance $x$ ahead of initial crack tip, (b) traction-free crack extension $\Delta a_{\mathrm{f}}$, extent of damage zone $\Delta a_{\mathrm{D}}$, and extent of plastic zone size $r_{\mathrm{P}}$ versus inferred crack extension $\Delta a_{\mathrm{PD}}$. Extent of crack bridging for $\bar{\rho}=9.6 \%$ : (c) $f$ versus distance $x$ ahead of initial crack tip, (d) $\Delta a_{\mathrm{f}}, \Delta a_{\mathrm{D}}$, and $r_{\mathrm{P}}$ versus $\Delta a_{\mathrm{PD}} \ldots \ldots \ldots \ldots$

8 Geometry of the SENB specimen used in FE simulations and details of the notch tip mesh. . . . . . . . . . . . . . . .

9 (a) Idealised true stress versus true strain response for the foam of $\bar{\rho}=6.6 \%$.

(b) Comparison of the predicted and measured load versus displacement response of the bend specimen (without a pre-notch). . . . . . . . . . .

10 (a) Assumed traction $T$ versus separation $\delta$ response for the cohesive zone. (b) Predictions of the load versus displacement response of a deeply notched bend specimen with the 3 limiting cases of cohesive properties: (i) $\hat{\sigma}=\Gamma_{0}=\infty$, (ii) rigid, perfectly plastic cohesive zone $\left(\hat{\sigma}=\sigma_{Y}, \Gamma_{0}=\infty\right)$, and (iii) $\hat{\sigma}=\sigma_{Y}, \Gamma_{0} \rightarrow$ 0 . The measured mean response for $\bar{\rho}=6.6 \%$ is included. . . . . . . . . .

11 (a) Contours of goodness-of-fit $\chi$, and (b) cohesive laws for the 3 best fitting

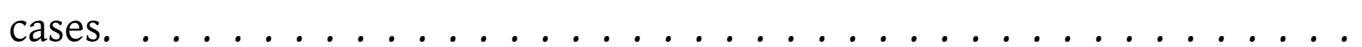

12 Comparison of the FE predictions for cases A, B, and C labelled in Fig. 11(a) with experimental observations for $\bar{\rho}=6.6 \%$ : (a) load $P$ versus displacement $v$ response, $(\mathbf{b})$ evolution of crack tip opening displacement $\left(\delta_{\mathrm{T}}\right)$ and crack mouth opening displacement $\left(\delta_{\mathrm{M}}\right)$ with $v$, (c) extent of the damage/bridging zone $\Delta a_{\mathrm{D}}$ versus displacement $v$, (d) traction-free crack extension $\Delta a_{\mathrm{f}}$ versus $v$, (e) $\Delta a_{\mathrm{D}}$ versus $\Delta a_{\mathrm{f}}$, and (f) relative extent of the plastic zone $r_{\mathrm{P}}$ to the damage zone (with $\mathrm{O}$ denoting the point of initiation of a traction-free crack,

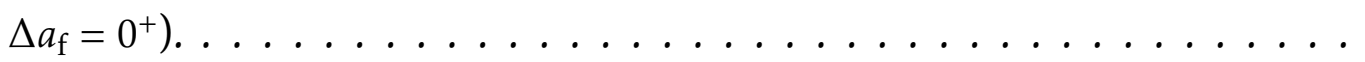


13 Response of a double edge notch specimen under tension: (a) geometry and loading, (b) measured and predicted load versus displacement response. The cohesive traction $T$ versus opening $\delta$ response for the best fitting case, case B, of the bend toughness test is included. . . . . . . . . . . . 37

14 Comparison of the displacement contours from DIC and FE simulations for (a) $a=15 \mathrm{~mm}$ and (b) $a=20 \mathrm{~mm}$ at peak load. (c) FE contours of strain in the loading direction for the two notch geometries, also at peak load. . . . . . . . 38 


\section{Figures}

(a)

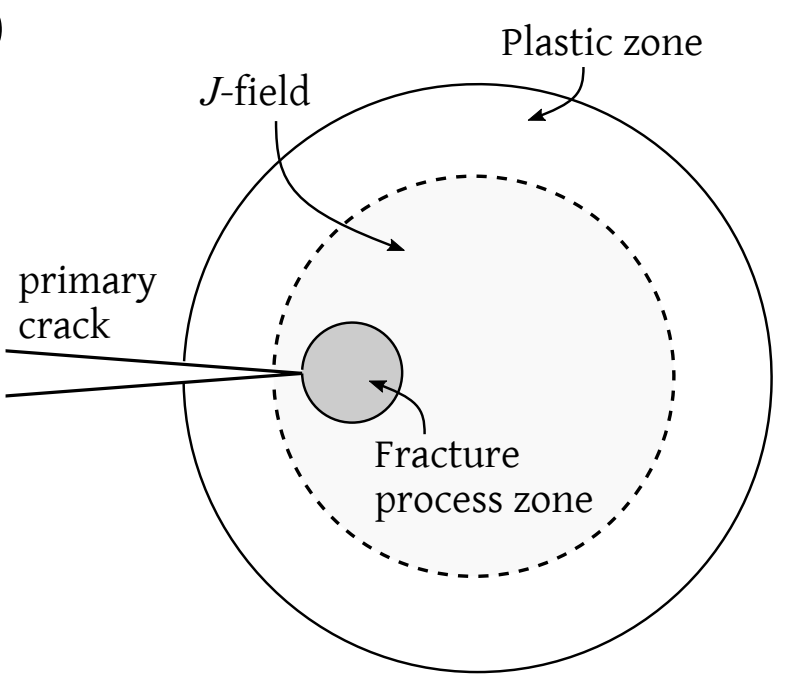

(b)

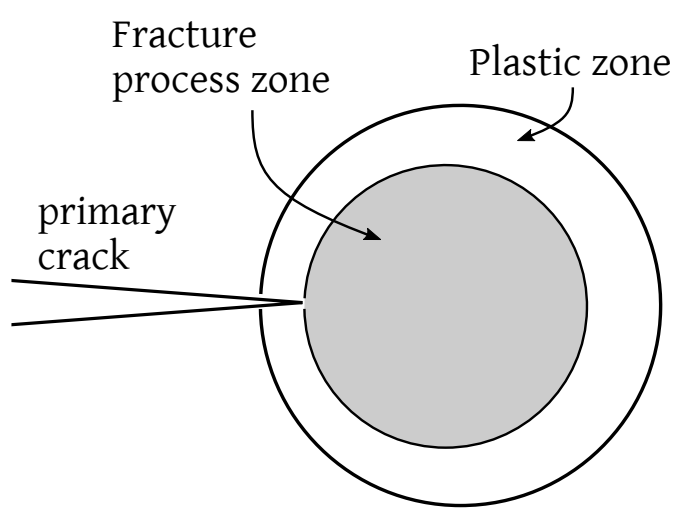

Fig. 1. (a) A fracture process zone (FPZ) embedded within a crack tip J-field; (b) no crack tip J-field is present due to the existence of a FPZ comparable in dimension to the plastic zone size.

(a)

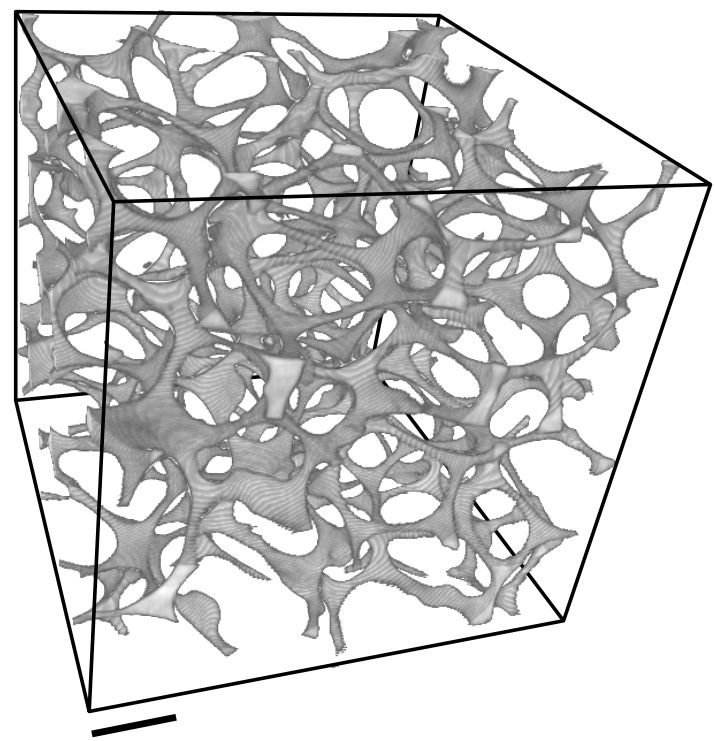

(b)

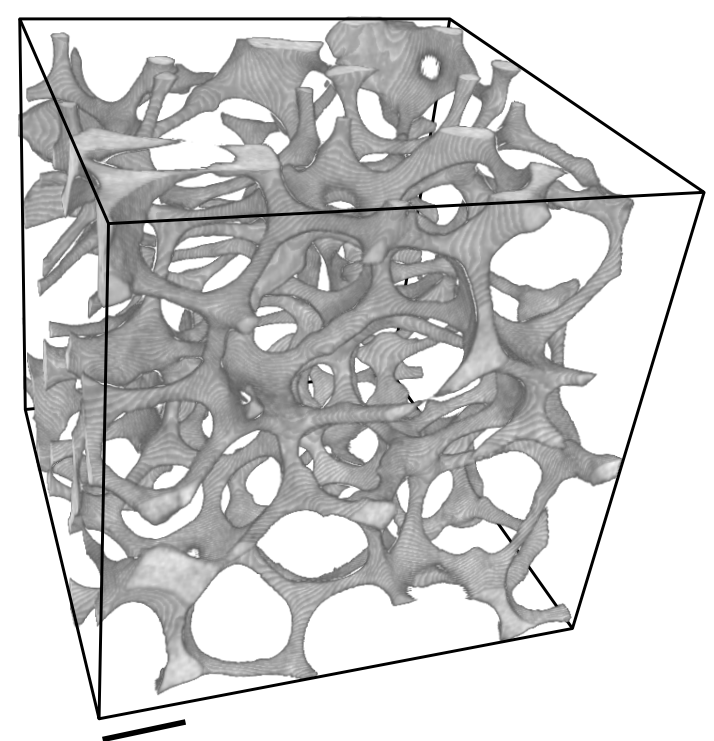

Fig. 2. X-ray CT images of aluminium alloy foam of (a) relative density $\bar{\rho}=6.6 \%$ and (b) $\bar{\rho}=9.6 \%$. The scale bar is of length $1 \mathrm{~mm}$. 
(a)

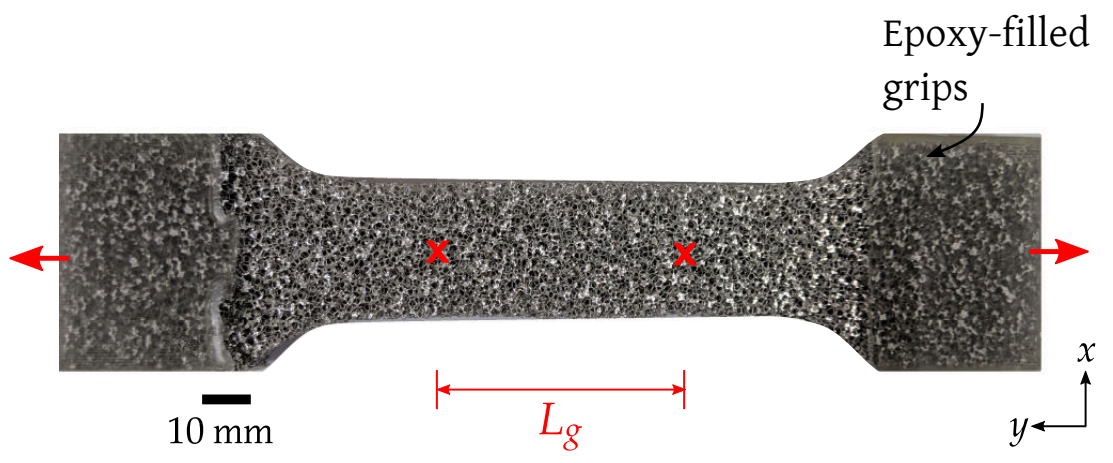

(b)

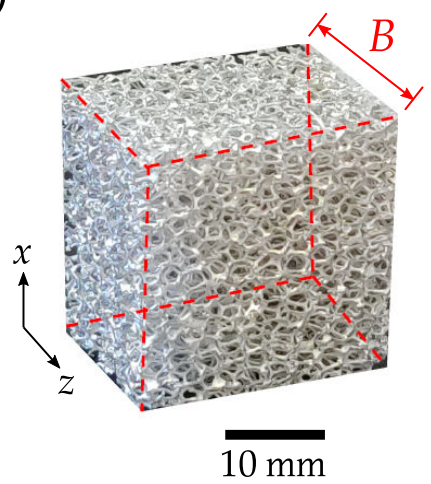

(c)

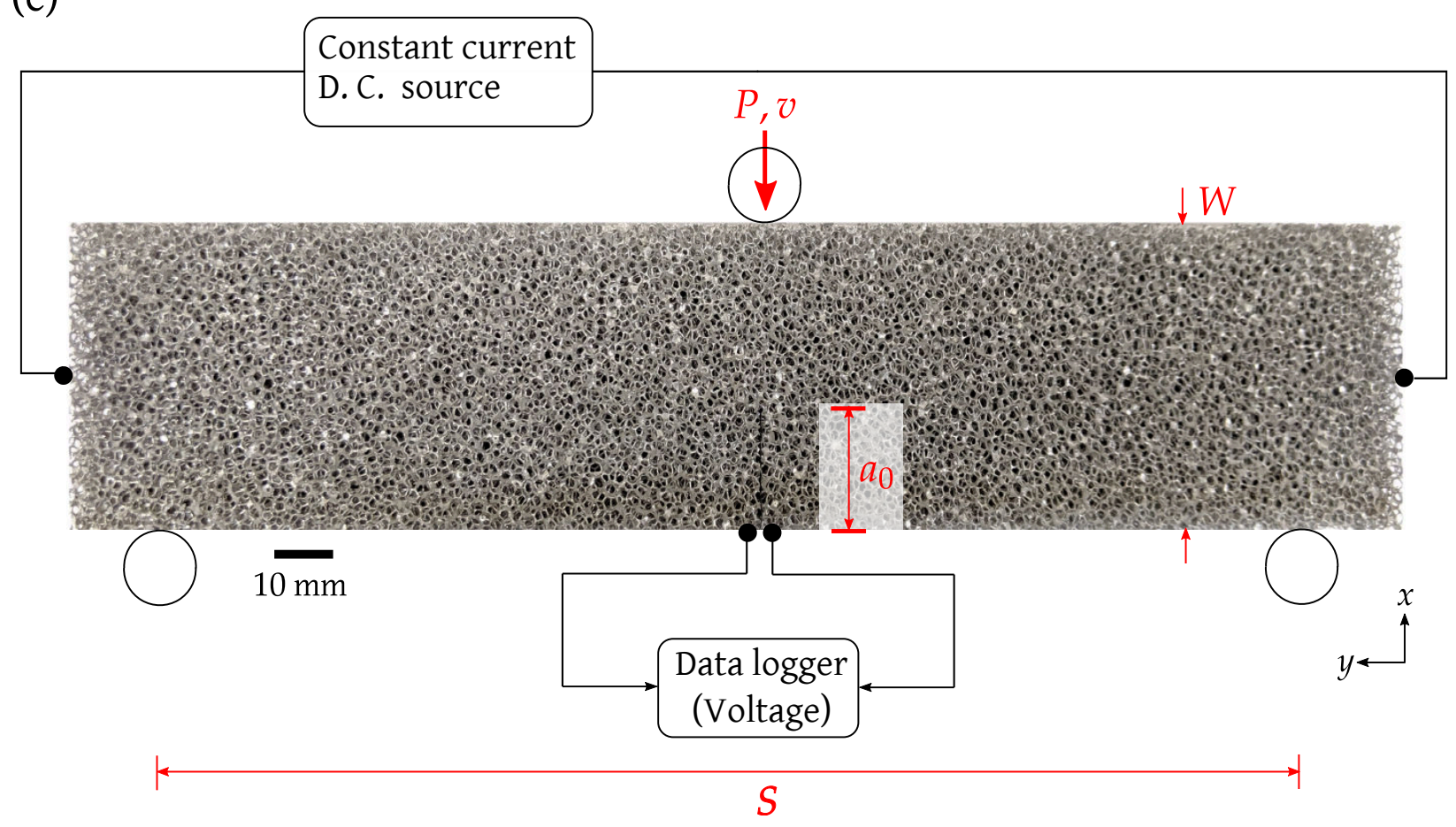

(d)

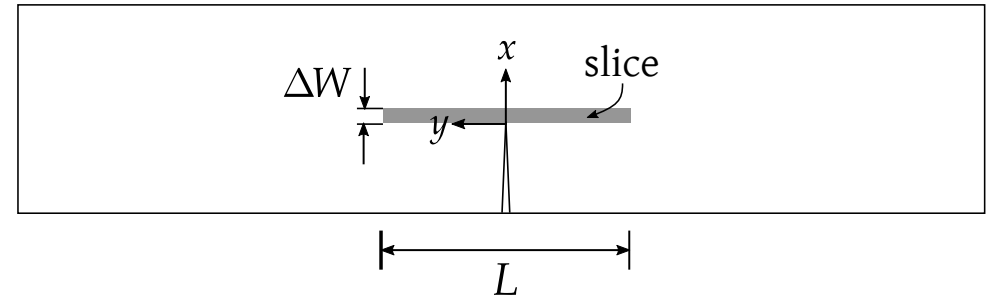

Fig. 3. Specimen geometries for (a) uniaxial tension test, (b) uniaxial compression test, and (c) fracture toughness test along with the apparatus. The out-of-plane thickness of all specimens is $B=26.4 \mathrm{~mm}$. (d) Definition of a slice used in the XCT analysis for damage visualisation. 


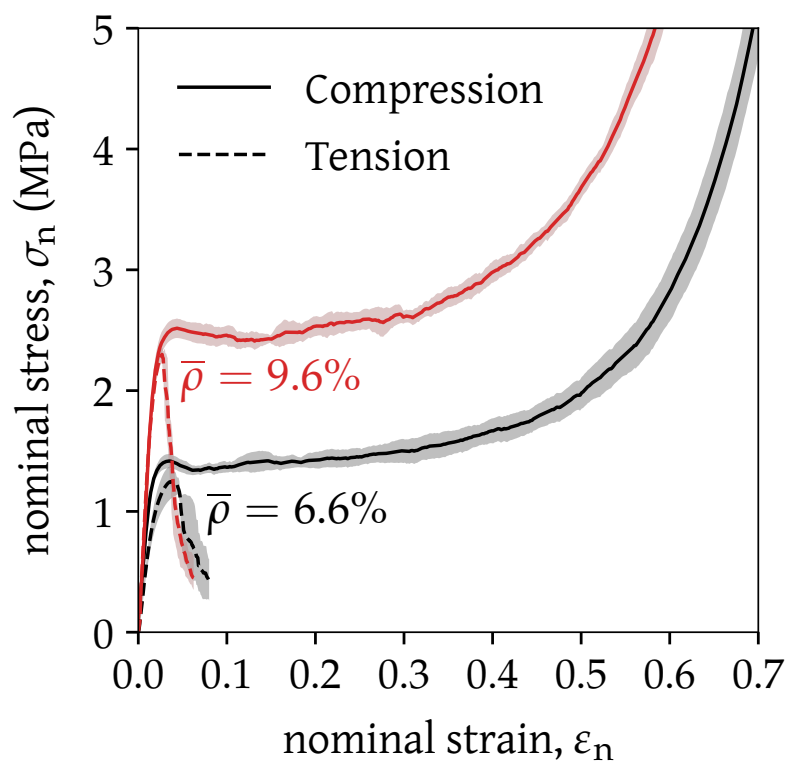

Fig. 4. Uniaxial tension, and in-plane compression, stress versus strain curves for the foams. 

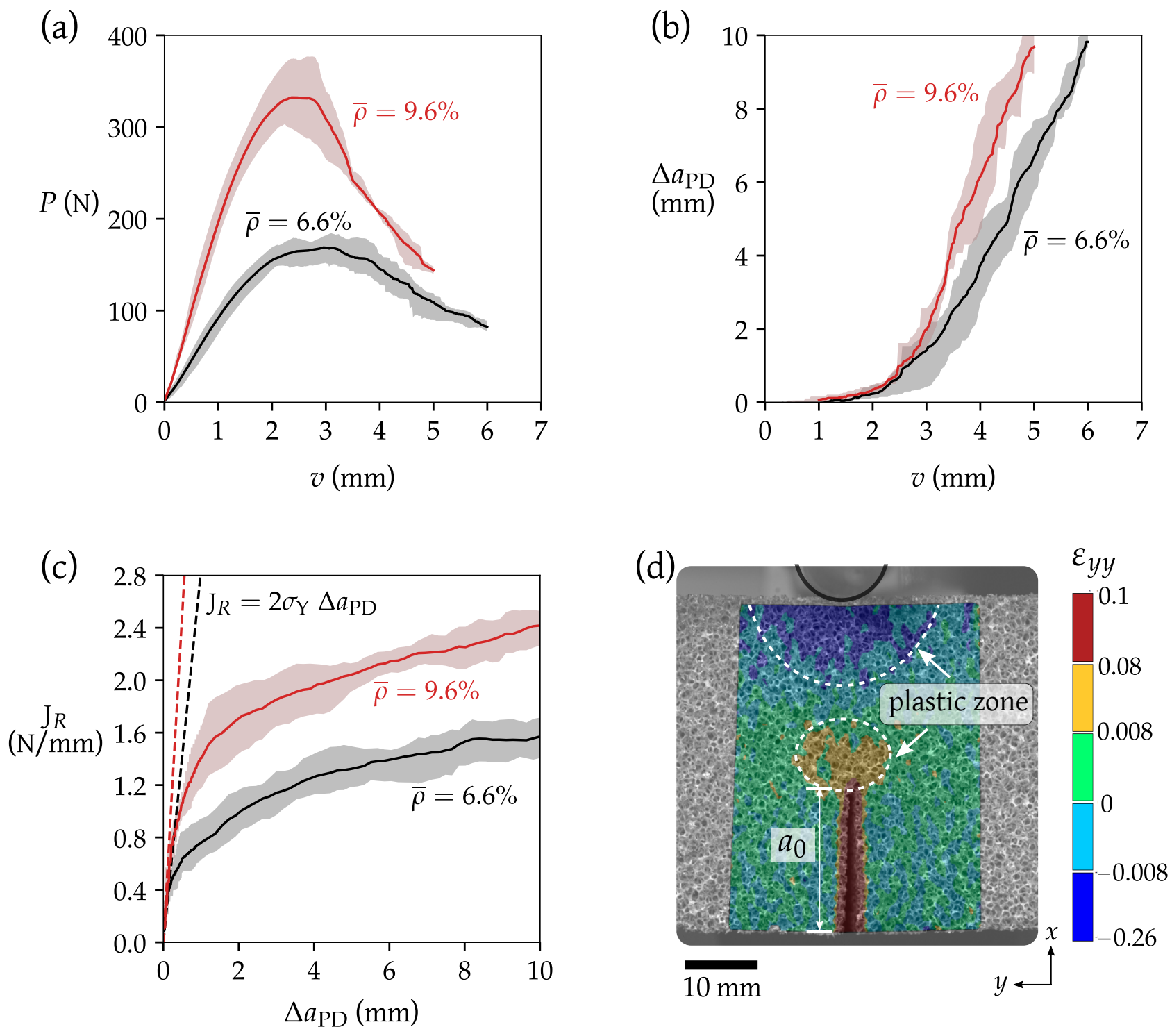

Fig. 5. Fracture response of deep-notched bend specimens: (a) Load $P$ versus cross-head displacement $v$ response, (b) crack extension $\triangle a_{\mathrm{PD}}$, as measured from the DCPD method, versus $v$, (c) crack growth resistance curves for $\bar{\rho}=6.6 \%$ and $9.6 \%$ specimens, and (d) DIC contours of longitudinal strain $\varepsilon_{y y}$ in a specimen of $\bar{\rho}=6.6 \%$ at peak load. The yield strain of this foam is $\varepsilon_{Y}=\sigma_{Y} / E=0.008$. 

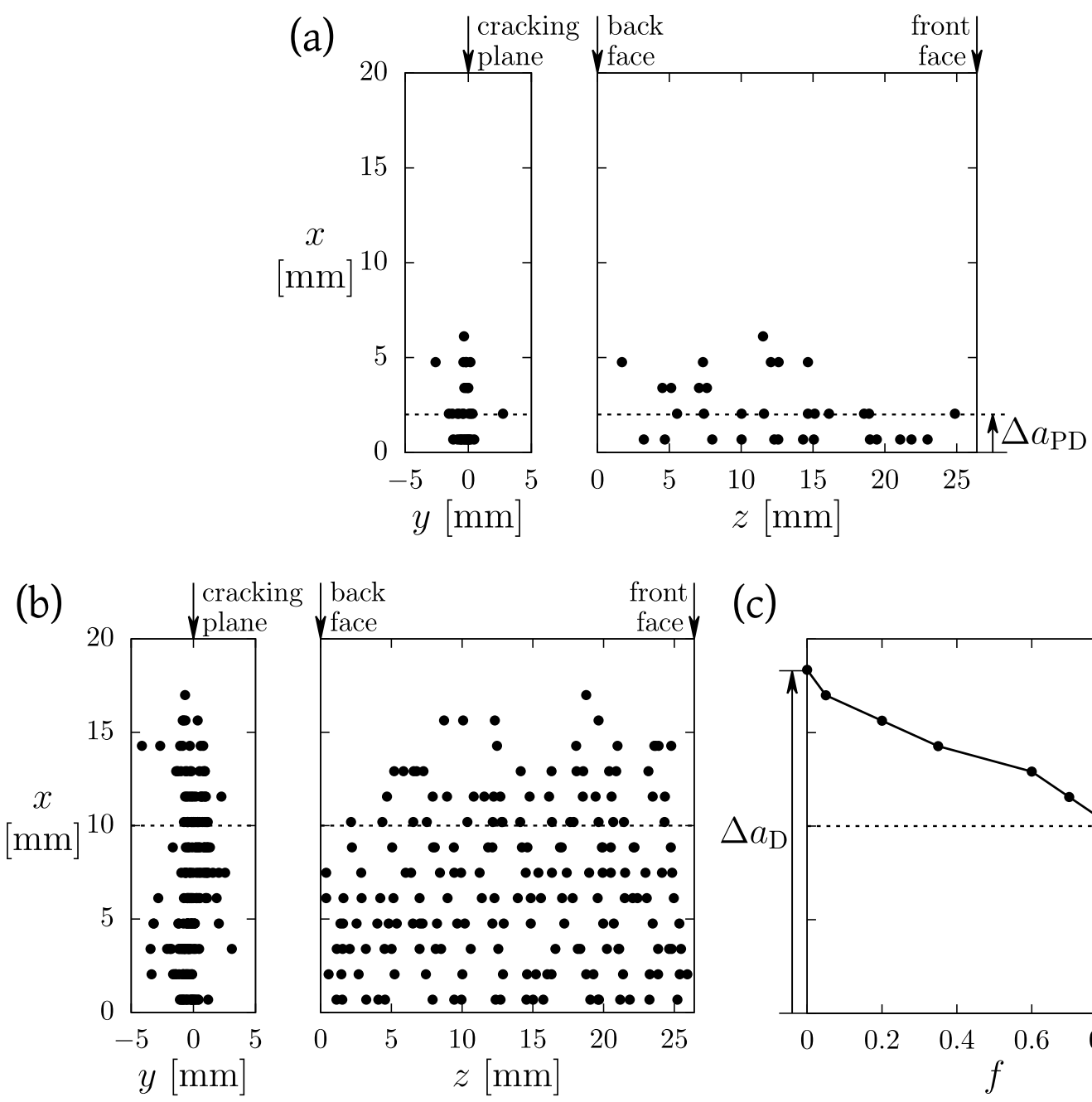

(c)

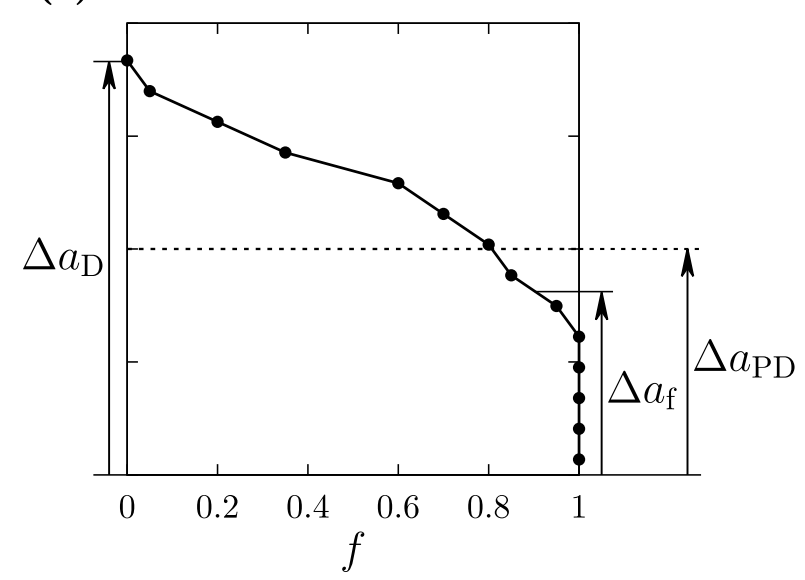

Fig. 6. Distribution of failed struts as determined from XCT analysis in a representative specimen of $\bar{\rho}=6.6 \%$ : (a) Location of failed struts for $\Delta a_{\mathrm{PD}}=2 \mathrm{~mm}$ : the projected view in the $(x, y)$ plane shows failed struts over all $z$; likewise, the view in the $(x, z)$ plane shows failed struts over all $y$-values. (b) Location of failed struts for $\Delta a_{\mathrm{PD}}=10 \mathrm{~mm}$ : again, the projected view in the $(x, y)$ is over all $z$; likewise, the view in the $(x, z)$ plane is over all $y$. (c) fraction of failed struts $f$ along the ligament ( $x$-direction) for $\Delta a_{\mathrm{PD}}=10 \mathrm{~mm}$. 
(a)

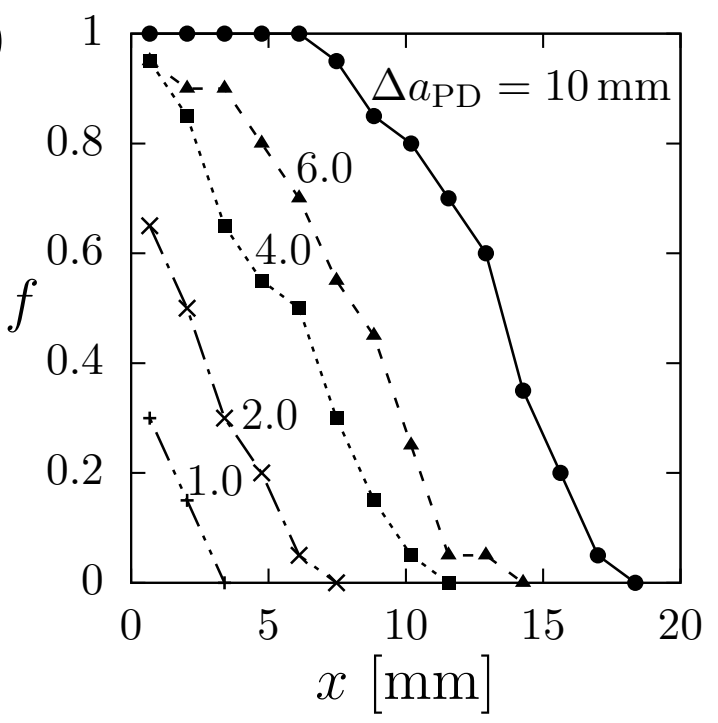

(c)

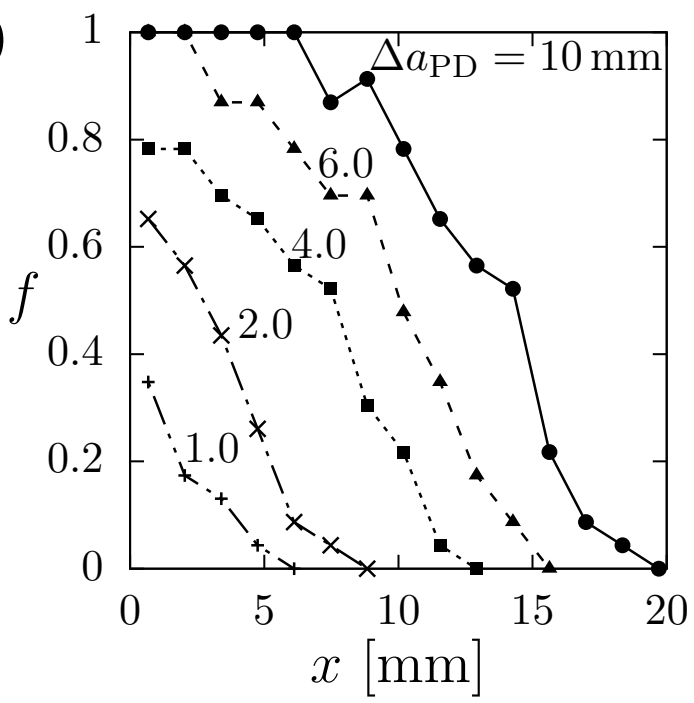

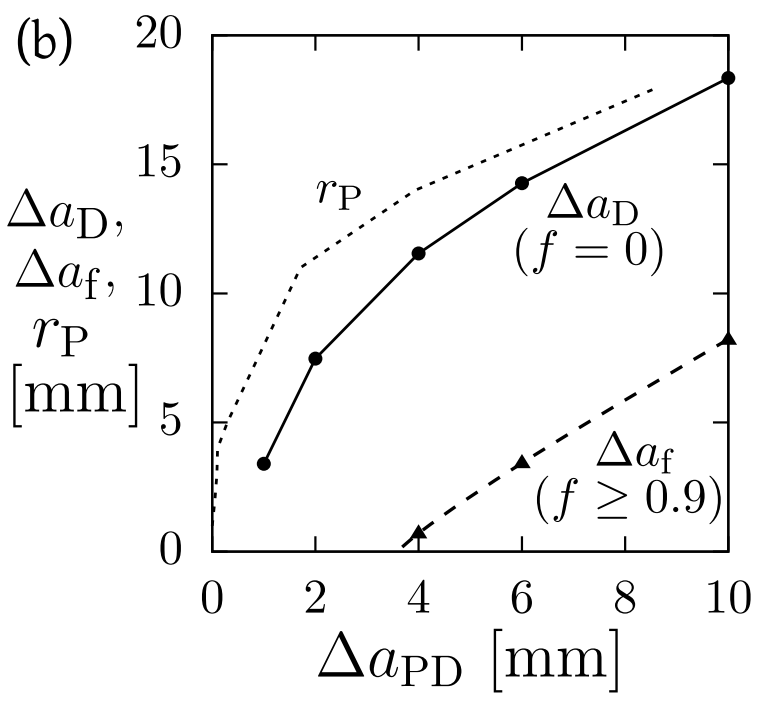

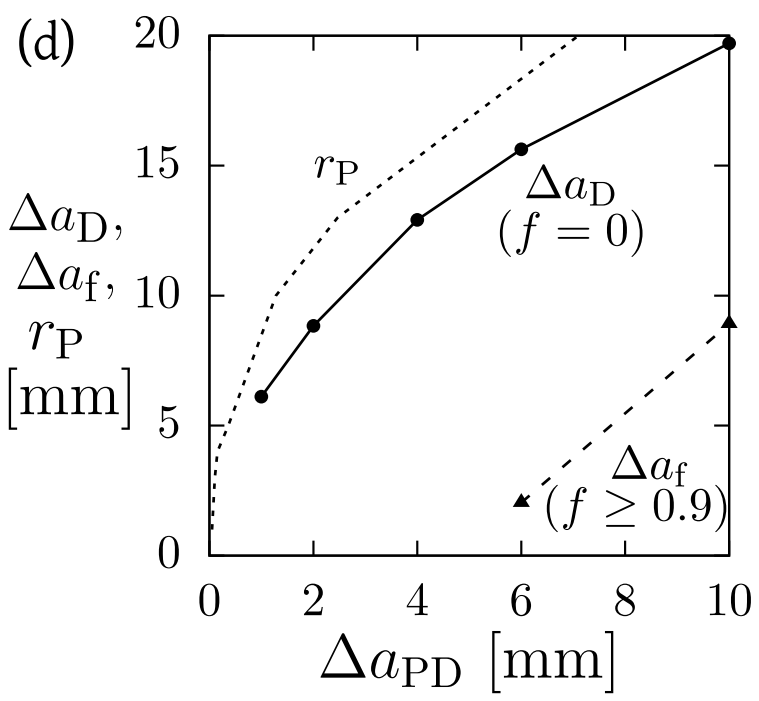

Fig. 7. Extent of crack bridging for $\bar{\rho}=6.6 \%$ : (a) $f$ versus distance $x$ ahead of initial crack tip, (b) tractionfree crack extension $\Delta a_{\mathrm{f}}$, extent of damage zone $\Delta a_{\mathrm{D}}$, and extent of plastic zone size $r_{\mathrm{P}}$ versus inferred crack extension $\Delta a_{\mathrm{PD}}$. Extent of crack bridging for $\bar{\rho}=9.6 \%$ : (c) $f$ versus distance $x$ ahead of initial crack tip, (d) $\Delta a_{\mathrm{f}}, \Delta a_{\mathrm{D}}$, and $r_{\mathrm{P}}$ versus $\Delta a_{\mathrm{PD}}$. 


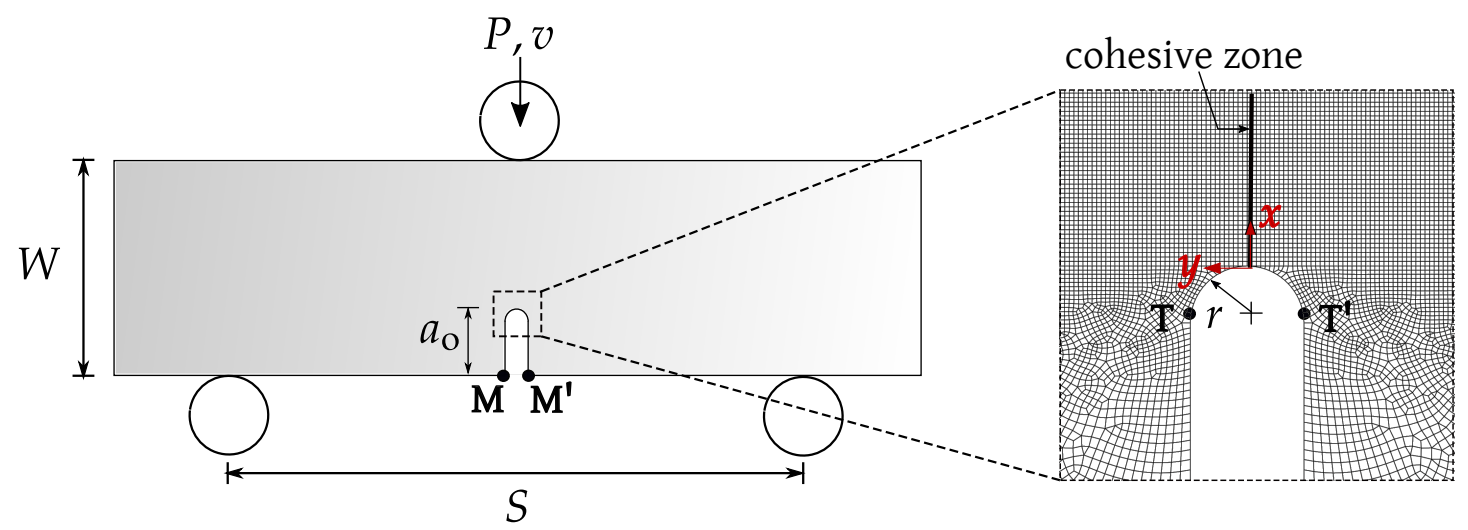

Fig. 8. Geometry of the SENB specimen used in FE simulations and details of the notch tip mesh.
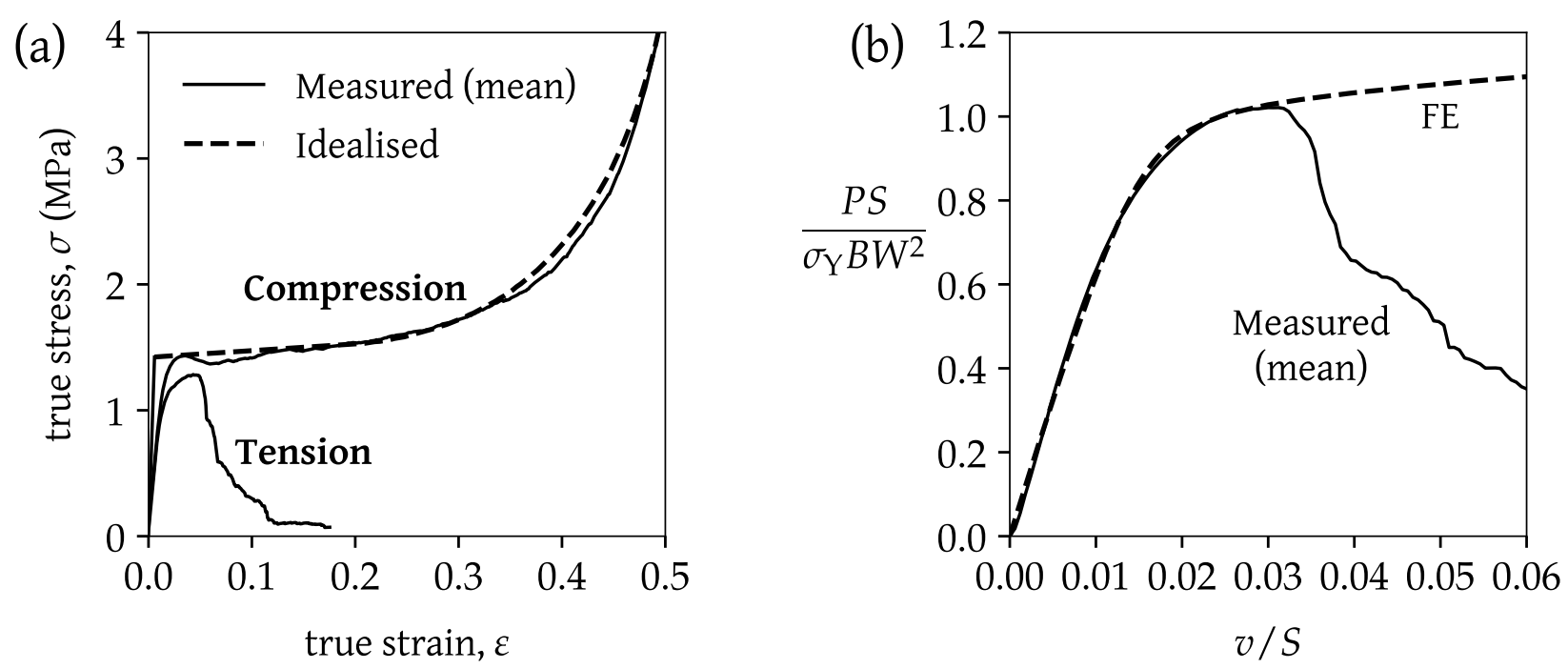

Fig. 9. (a) Idealised true stress versus true strain response for the foam of $\bar{\rho}=6.6 \%$. (b) Comparison of the predicted and measured load versus displacement response of the bend specimen (without a pre-notch). 
(a)

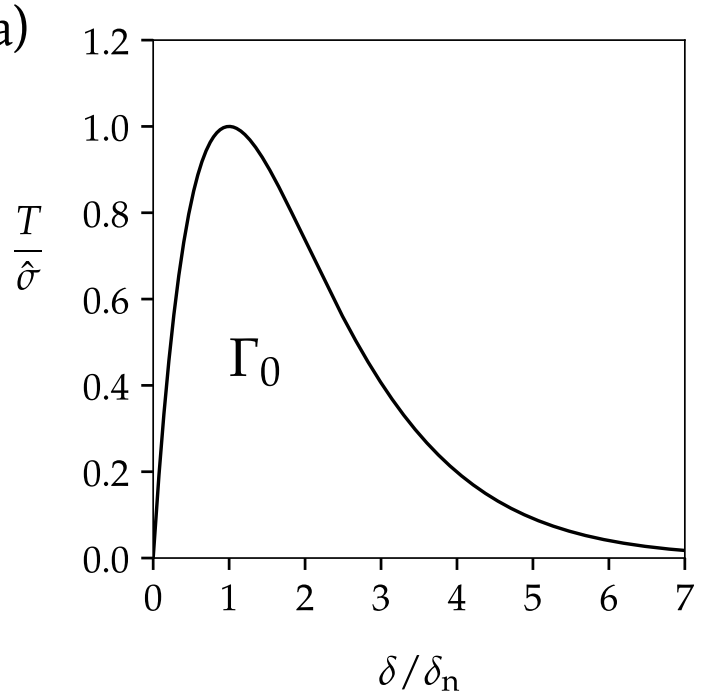

(b)

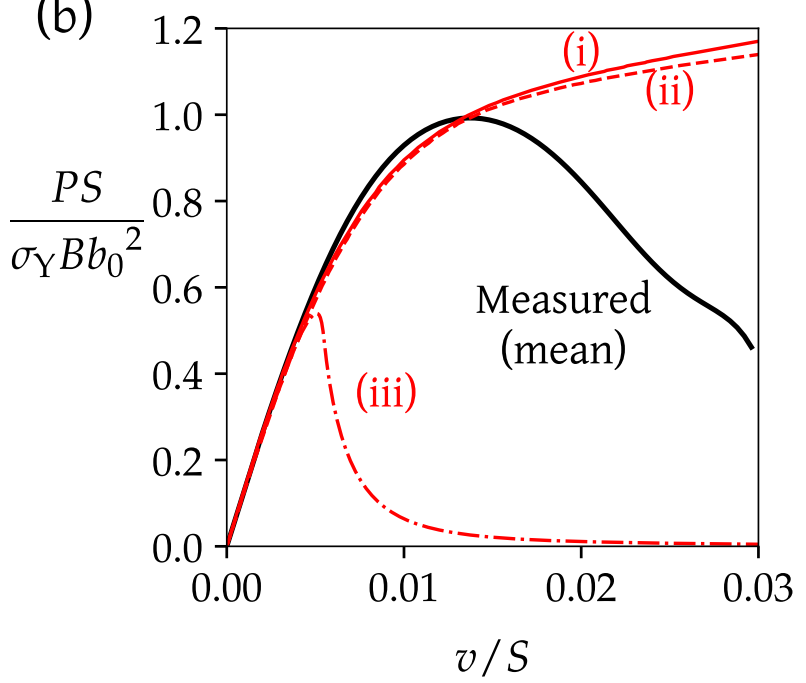

Fig. 10. (a) Assumed traction $T$ versus separation $\delta$ response for the cohesive zone. (b) Predictions of the load versus displacement response of a deeply notched bend specimen with the 3 limiting cases of cohesive properties: (i) $\hat{\sigma}=\Gamma_{0}=\infty$, (ii) rigid, perfectly plastic cohesive zone $\left(\hat{\sigma}=\sigma_{\mathrm{Y}}, \Gamma_{0}=\infty\right)$, and (iii) $\hat{\sigma}=\sigma_{\mathrm{Y}}, \Gamma_{0} \rightarrow 0$. The measured mean response for $\bar{\rho}=6.6 \%$ is included.
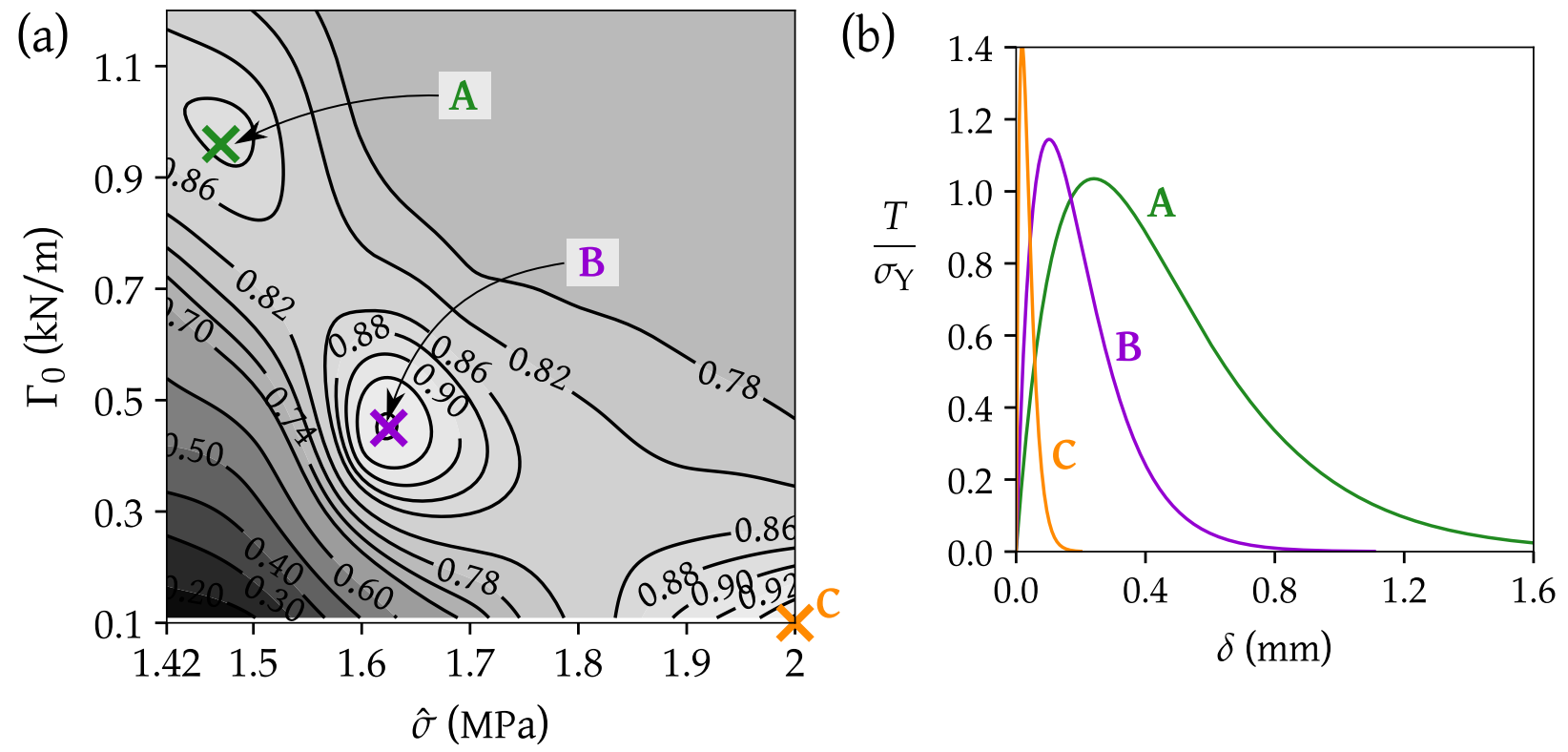

Fig. 11. (a) Contours of goodness-of-fit $\chi$, and (b) cohesive laws for the 3 best fitting cases. 

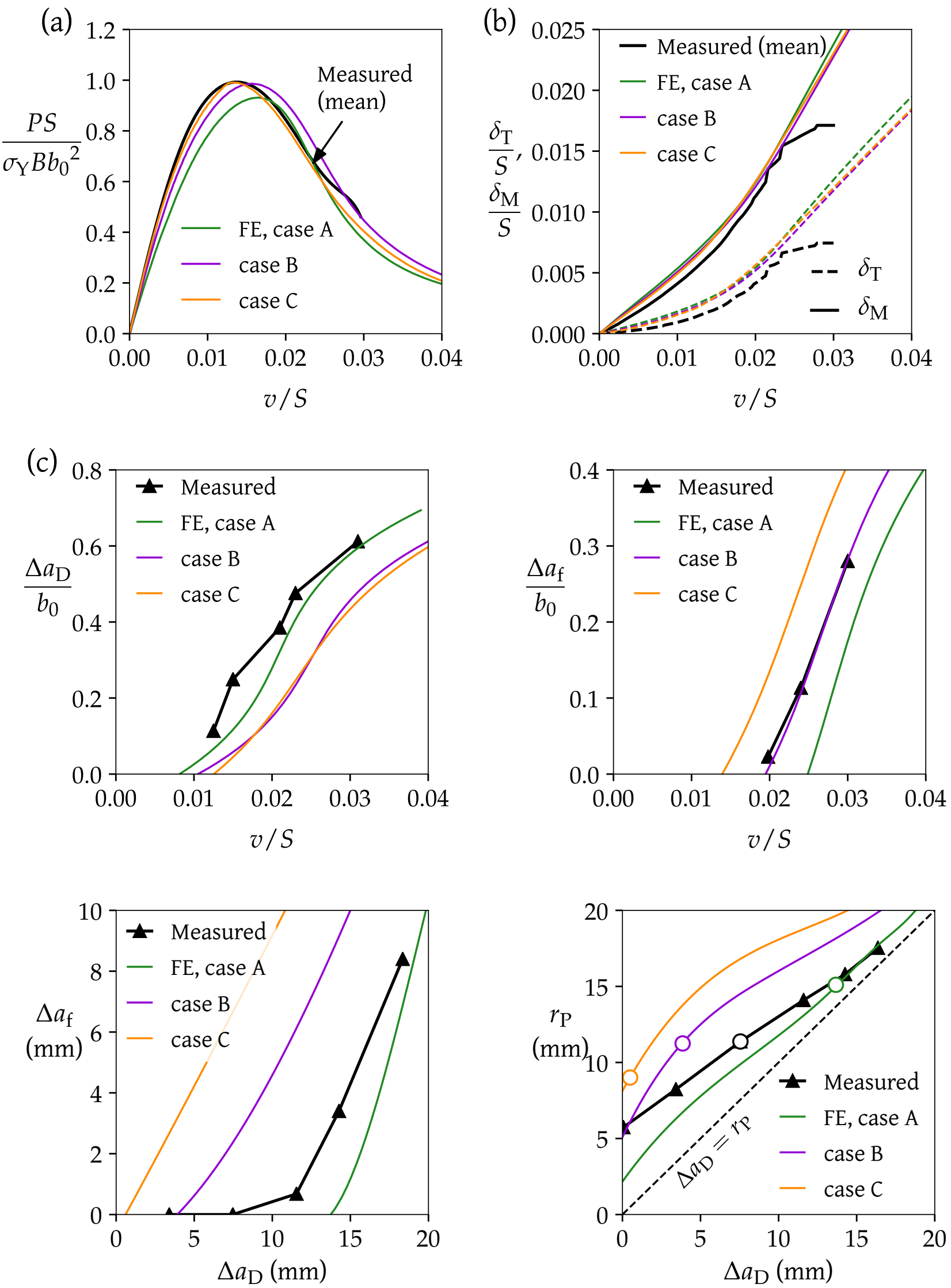

Fig. 12. Comparison of the FE predictions for cases A, B, and C labelled in Fig. 11(a) with experimental observations for $\bar{\rho}=6.6 \%$ : (a) load $P$ versus displacement $v$ response, (b) evolution of crack tip opening displacement $\left(\delta_{\mathrm{T}}\right)$ and crack mouth opening displacement $\left(\delta_{\mathrm{M}}\right)$ with $v$, (c) extent of the damage/bridging zone $\Delta a_{\mathrm{D}}$ versus displacement $v,(\mathrm{~d})$ traction-free crack extension $\Delta a_{\mathrm{f}}$ versus $v$, (e) $\Delta a_{\mathrm{D}}$ versus $\Delta a_{\mathrm{f}}$, and (f) relative extent of the plastic zone $r_{\mathrm{P}}$ to the damage zone (with $\mathrm{O}$ denoting the point of initiation of a traction-free $\mathrm{crack}, \Delta a_{\mathrm{f}}=0^{+}$). 
(a)

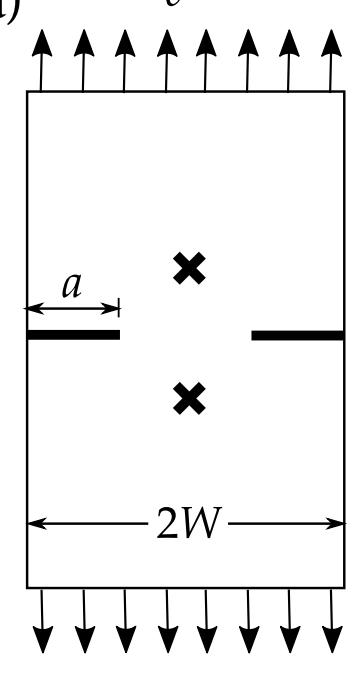

(b)

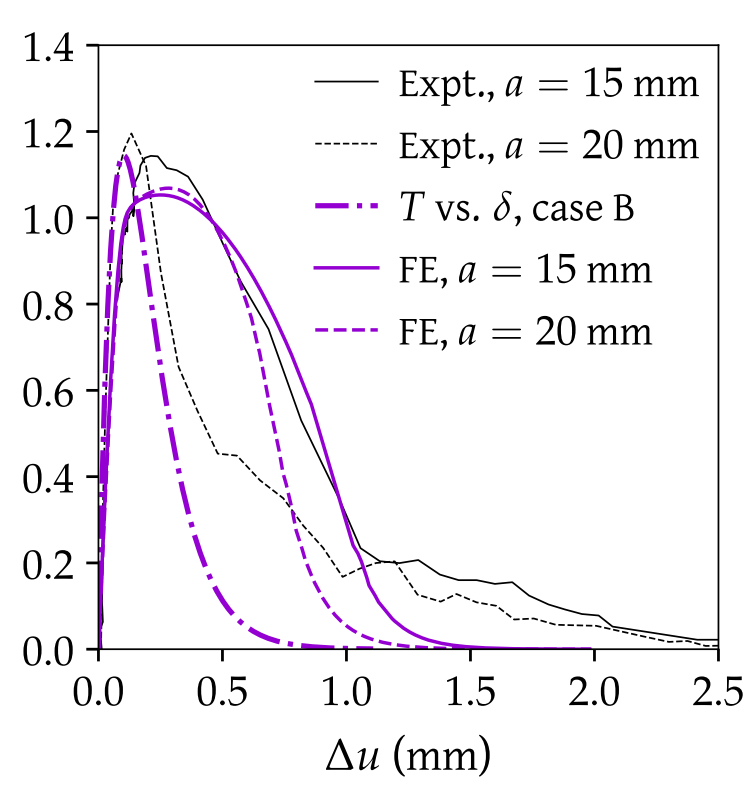

Fig. 13. Response of a double edge notch specimen under tension: (a) geometry and loading, (b) measured and predicted load versus displacement response. The cohesive traction $T$ versus opening $\delta$ response for the best fitting case, case B, of the bend toughness test is included. 
(a)

$a=15 \mathrm{~mm}$
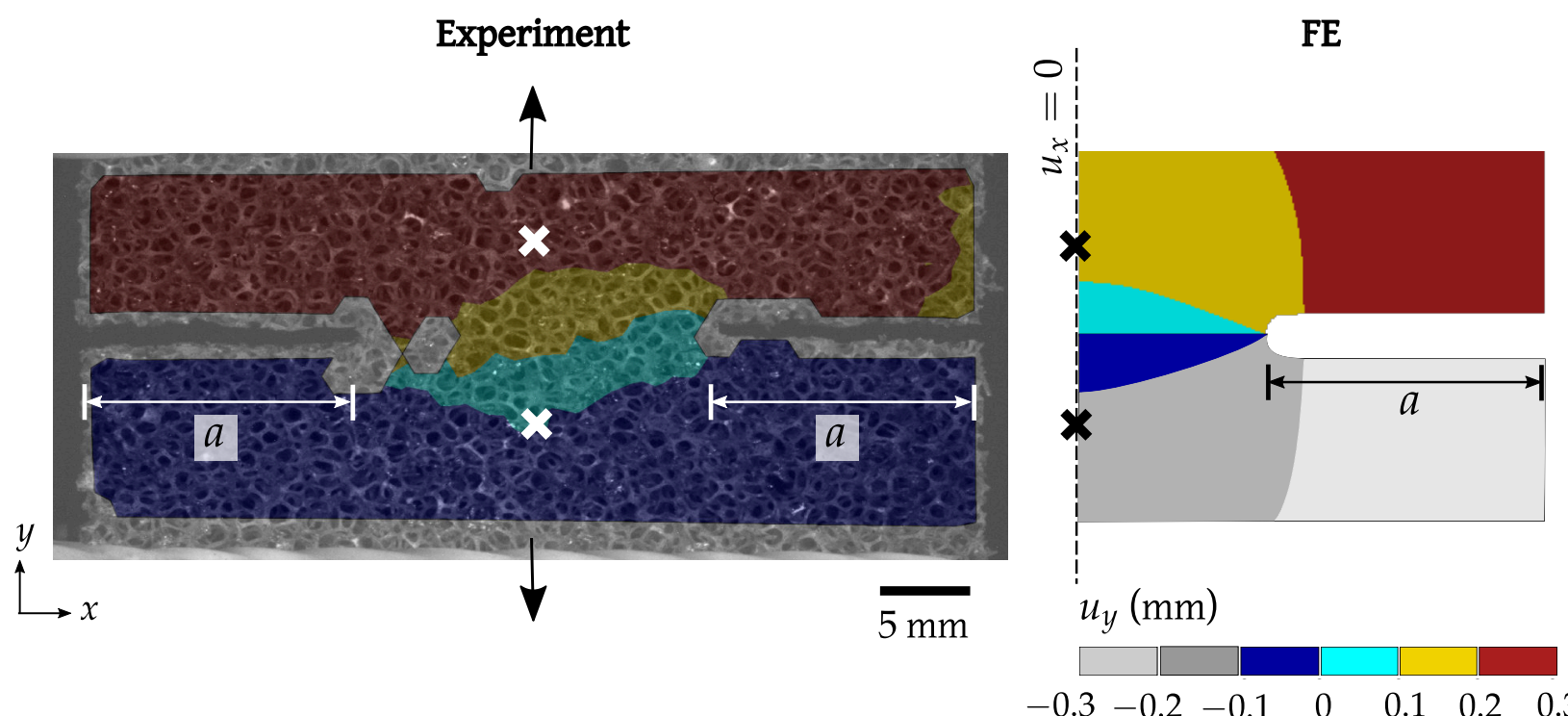

(b) $a=20 \mathrm{~mm}$
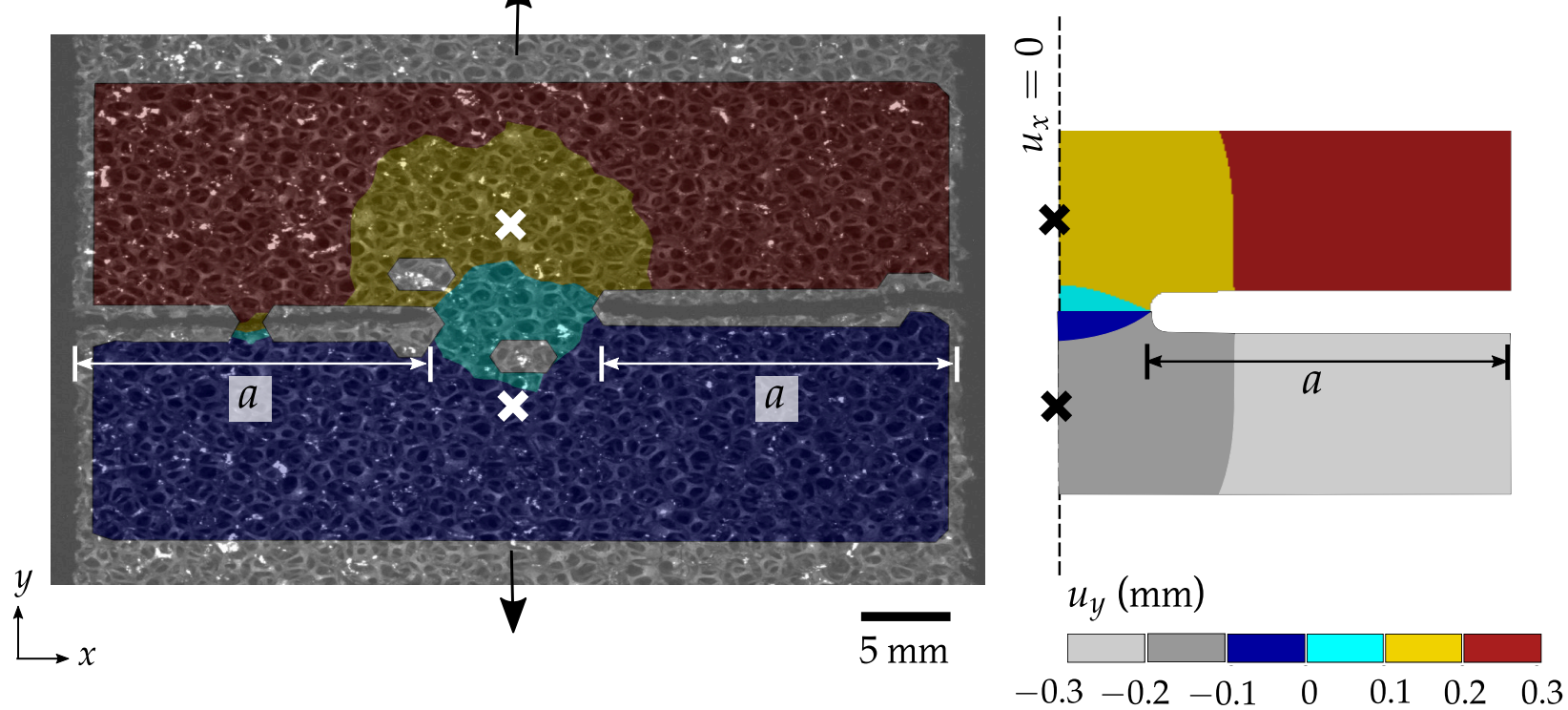

(c) $\mathrm{FE}$
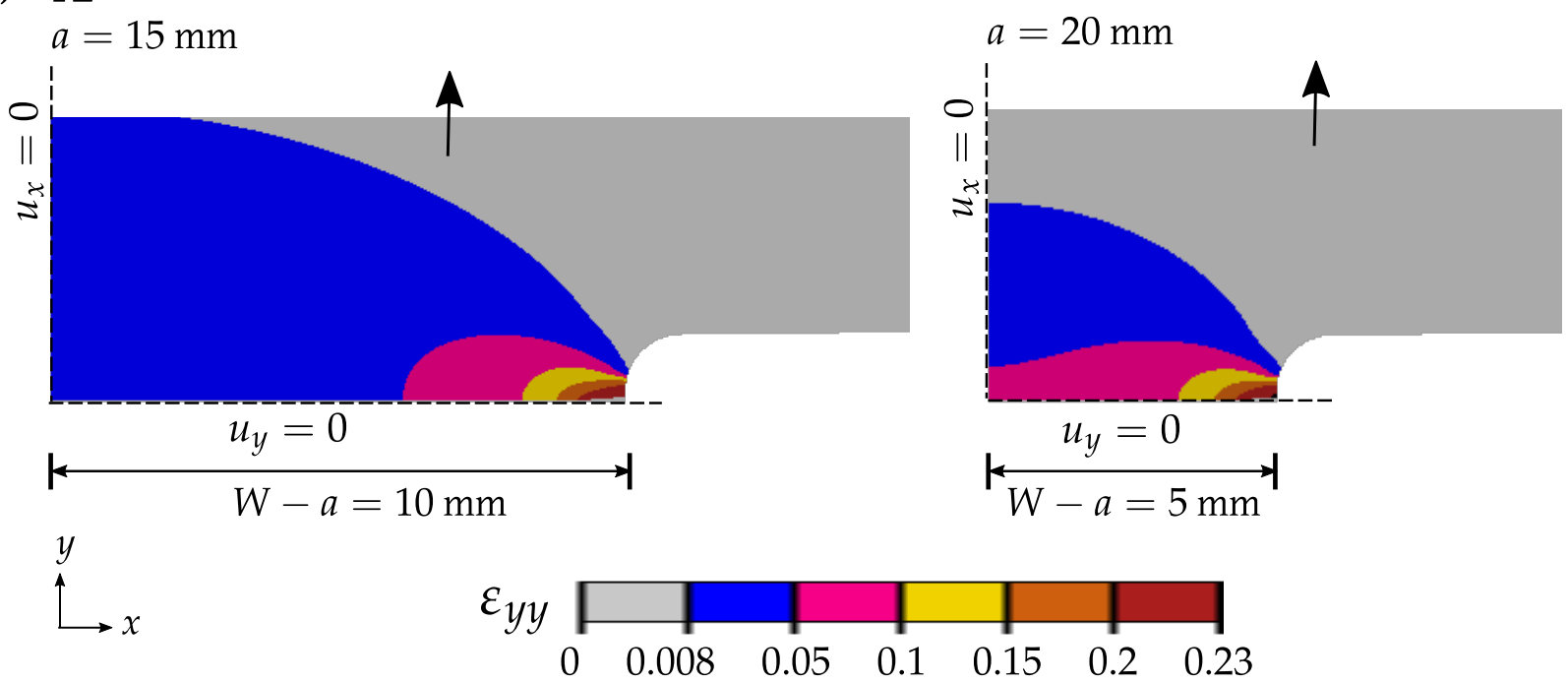

Fig. 14. Comparison of the displacement contours from DIC and FE simulations for (a) $a=15 \mathrm{~mm}$ and (b) $a=20 \mathrm{~mm}$ at peak load. (c) FE contours of strain in the loading direction for the two notch geometries, also at peak load. 